\title{
TESTIMONIANZE DI EDIFICI SACRI DI EPOCA CAROLINGIA E OTTONIANA NELL'ALTA VALLE DELL'ADIGE. GLI SCAVI DI CASTEL TIROLO
}

\author{
LORENZO DAL RI
}

UDC: 904(450):726

Original scientific paper

Manuscript received: 17. 02. 1997.

Revised manuscript accepted: 01. 04. 1997.
L. Dal Ri Soprintendenza per i beni culturali Ufficio beni archeologici

Bolzano Italia

L'attività di scavo della Soprintendenza Provinciale dell'Alto Adige ha avuto come oggetto nell'ultimo decennio alcuni edifici sacri che hanno restituito testimonianze riguardanti il periodo carolingio. Ciò vale in particolare per lo scavo di recente condotto a Castel Tirolo presso Merano, la casa d'origine della dinastia dei conti di Tirolo. Gli scavi hanno portato in luce $i$ resti di unantica chiesa di cui si era perduto ogni ricordo. La presenza di un sistema di tre absidi inserite nello spessore del muro orientale ha permesso di ricondurre questo edificio al tipo rätische Dreiabsidenkirchen . L'edificio risulta essere stato demolito ed interrato, probabilmente come conseguenza della costruzione del castello dotato di una sua propria capella (San Pancrazio), nell'XII sec. La prosecuzione degli scavi all'interno della chiesa ha mostrato come l'edificio carolingio insista sui resti di un edificio monoabsidato del V-VI sec., del quale è stato possibile riconoscere, murato e sigillato in antico, il Reliquiengrab con capsella in marmo e portareliquie intatto d'argento dorato.

Nel 1981 si tenne a Bolzano su iniziativa di N. Rasmo un convegno sull' arte carolingia ${ }^{1}$ in Alto Adige che nella storia locale degli studi può esser preso come un confine, tra due fasi di attività sostanzialmente diverse. In una prima (negli ultimi decenni del secolo scorso con P. Orsi, nel nostro secolo con J. Garber, G. Gerola, C. Cecchelli, E. Egg, Rasmo stesso) erano prevalse approfondite analisi storico-artistiche su evidenze (soprattutto sculture, poi stucchi ed affreschi) venute in luce in modo più o meno casuale, spesso nel corso di lavori di restauro. Sono degne di menzione ad es. le edizioni dei materiali di San Benedetto di Malles in Val Venosta, inoltre per il territorio trentino ad esempio taluni studi sui marmi architettonici di Stenico, di San Lorenzo di Tenno etc. ${ }^{2}$.

Con l'inizio degli anni ottanta si può far iniziare invece una nuova fase in cui si sono imposte come metodo di lavoro le indagini archeologiche sistematiche nel sottosuolo delle chiese. Nel 1981 sono state pubblicate le ricerche di R. Lunz nella chiesa di San Pietro di Quarazze-Gratsch presso Merano. Era poi in corso lo scavo al colle di Sabiona-Säben in Val d'Isarco, del quale i risultati sono stati resi noti in forma compendiaria nel $1986^{3}$.

In particolare poi negli ultimi 15 anni, soprattutto nel territorio altoatesino, le usuali attività di restauro e riassetto di chiese storiche hanno comportato in misura crescente la necessità di condurre indagini archeologiche che hanno reso una serie di testimonianze anche riguardo al periodo carolingio-ottoniano.

\section{LO SCAVO DI CASTEL TIROLO}

Nel settembre 1992 alcuni consistenti lavori all'esterno del muro di cinta meridionale di Castel Tirolo hanno portato in luce i resti di un antico edificio sepolto, di cui si era perduto ogni ricordo. Il luogo situato presso Merano nell'alta Valle dell'Adige, all'imbocco del tratto finale che prende il nome di Val Venosta, è noto perché vi sorge lo Stammschloß dei Conti di Tirolo i quali ebbero importanza determinante come artefici nel XIII secolo, soprattutto ad opera di Mainardo II, dell'entità geopolitica che da questa dinastia ha preso il nome, il Tirolo appunto.
In particolare furono notati alcuni tratti di un muro semicircolare con una struttura murata rettangolare al centro, in cui fu presto agevole riconoscere i resti di un'abside e rispettivamente di un altare ${ }^{4}$. Una serie di campagne di scavo (soprattutto 1994 e 1996) ha permesso di rico-

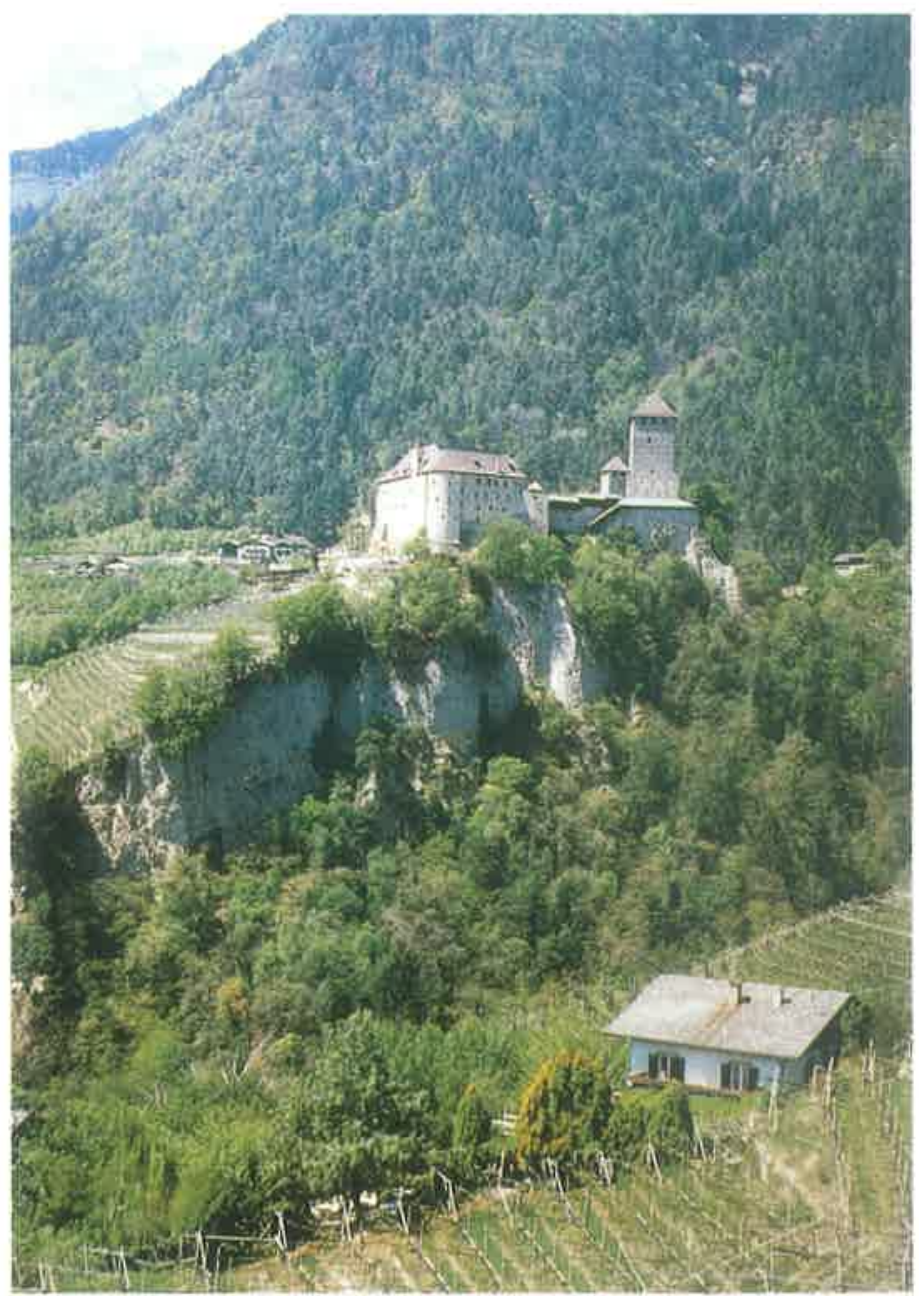

Fig. 1. Castel Tirolo presso Merano. Il colle del Castello (da Est). 

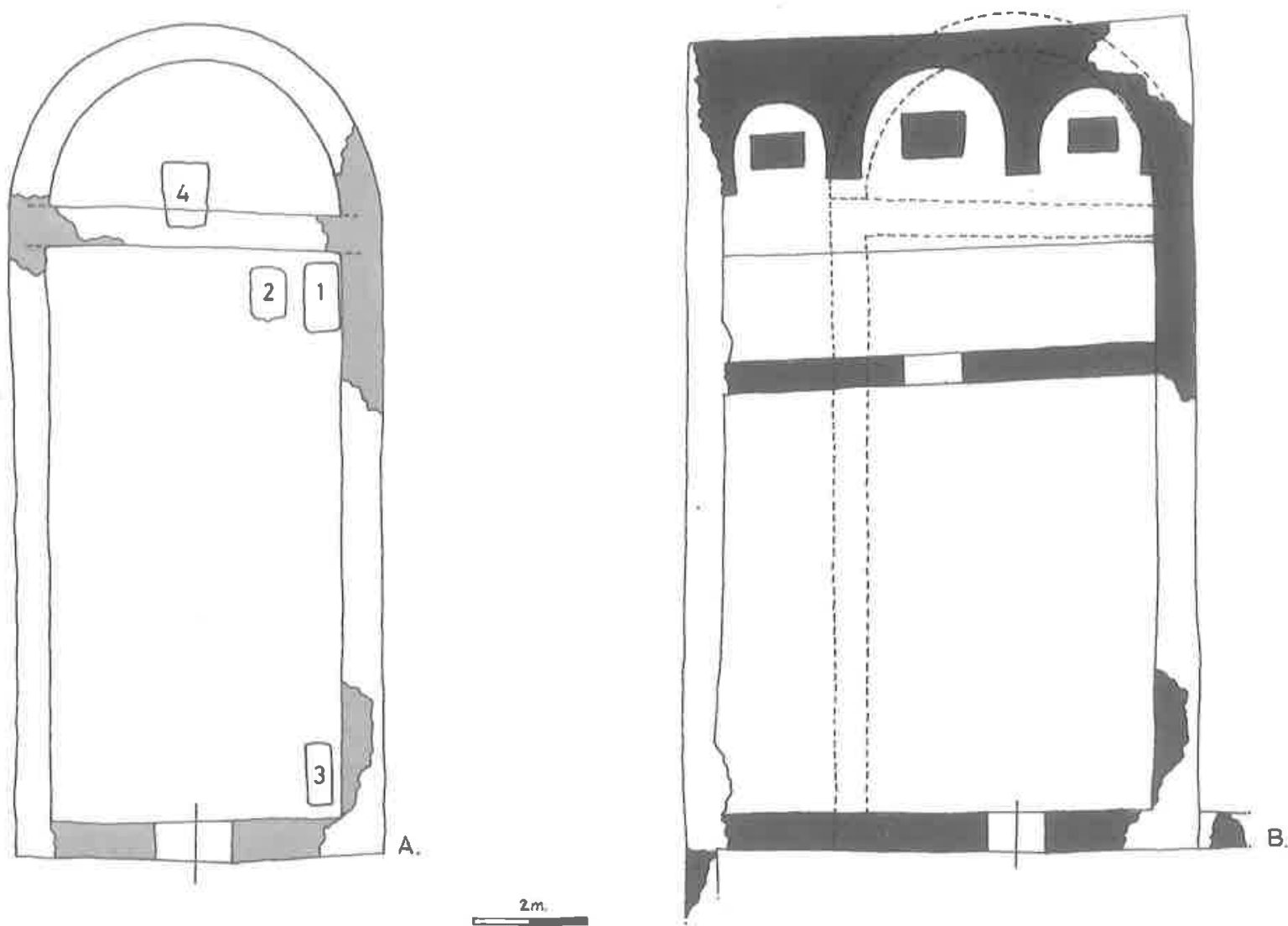

Fig. 2. Castel Tirolo presso Merano: a) ricostruzione del perimetro della chiesa paleocristiana con le tombe (1-3) ed il loculo per le reliquie (4); b) pianta semplificata degli scavi con indicati in viola la chiesa triabsidata, in punteggiato il perimetro della chiesa paleocristiana.

noscere nella collina su cui sorge il castello un'area archeologica di rilevante importanza. Ritrovamenti sporadici non erano mancati in precedenza ${ }^{5}$ ma non in misura tale da dare un quadro adeguato della situazione reale.

In particolare nell'ambito della chiesa di recente scoperta, uno dei primi risultati è stato l'accertamento nell'area del coro di un sistema formato da tre absidi, ciascuna dotata di un altare, collegate ad un'unica aula ${ }^{6}$. Tale edificio triabsidato misura $\mathrm{m} 14,5$ di lunghezza per $\mathrm{m} 9$ di larghezza. Le tre absidi sono completamente inserite nello spessore del muro orientale che verso l'esterno è rettilineo. Alcune parti dell'edificio dovettero avere una decorazione pittorica, ritrovata finora in modesti lacerti in uno strato di demolizione. Vi sono tracce di un recinto presbiteriale con base in muratura, con un'apertura nella parte mediana larga $\mathrm{m} 0,8$. Un gradino separa i tre altari in muratura dalla navata. L'ingresso è sul lato ovest. In occasione della costruzione di un muro di sostegno in cemento destinato a proteggere l'area della chiesa, furono poi riconosciute nel 1994 tracce dell'esistenza di un ambiente murato (di funzione non ancora chiarita) sul lato occidentale, dinanzi alla facciata. Il pavimento della chiesa carolingia, composto da tre livelli sucessivi di malta di calce era ancora presente per quanto profondamente alterato e fessurato su tutta l'estensione della navata, inoltre nel coro e nelle absidi. In esso, già al momento dello scoprimento, si disegnavano delle depressioni nettamente delimitate, interpretabili come cedimenti corrispondenti a tombe della fase precedente, ipotesi poi confermata nelle ricerche successive.

La vita di questo edificio non sembra aver trovato il suo epilogo in un episodio di distruzione o per meglio dire non si sono trovate palesi tracce di incendio (come uno strato uniforme di carboni, pietre e calce dei muri alterati dal calore, etc.).

Netti invece i segni di un dissesto nell'assetto statico dell'edificio a cui si è posto rimedio nel corso di un'articolata fase tarda di ricostruzione, la quale ha comportato adattamenti delle strutture murarie, soprattutto sul lato di valle, particolarmente interessato da cedimenti strutturali. Uno strato di terriccio artificialmente gettato è servito in questa fase a livellare il pavimento sconnesso. Una grossa buca presso il recinto del coro esattamente sull'asse della navata, è poi verosimilmente il residuo di un sistema di copertura in cui un massiccio pilastro ligneo centrale doveva sostenere un tetto di travi, queste ultime forse di recupero. Intatte rimasero comunque in questa fase le strutture dei tre altari nelle tre absidi (per cui si direbbe mantenuta la funzione di edificio sacro) Era invece scomparso il recinto presbiteriale. Difficile è valutare la durata e soprattutto il contesto storico di questa ricostruzione: in ogni caso questo edificio ebbe pur nella rozzezza delle tecniche architettoniche, strutture poderose ed in particolare il muro verso valle, legato con argilla, risulta essere stato largo non meno di $80 \mathrm{~cm}$.

$\mathrm{E}^{\prime}$ stato poi possibile rilevare chiare tracce nelle murature di una successiva demolizione sistematica, con evidente asporto delle pietre maggiori ancora utilizzabili. Proponiamo di porre in relazione questa ultima spoliazione con la costruzione del castello dei Conti di Tirolo per la gran parte ancora oggi esistente. Per quest'ultima possediamo una serie di dati dendrocronologici: in particolare un gruppo di tronchi da cui sono state ricavate le travi del palazzo meridionale fu abbattuto nel 1138-1139. Le medesime si riferiscono però già ad una fase di ristrutturazione mentre la costruzione originale potrebbe risalire almeno ad una 


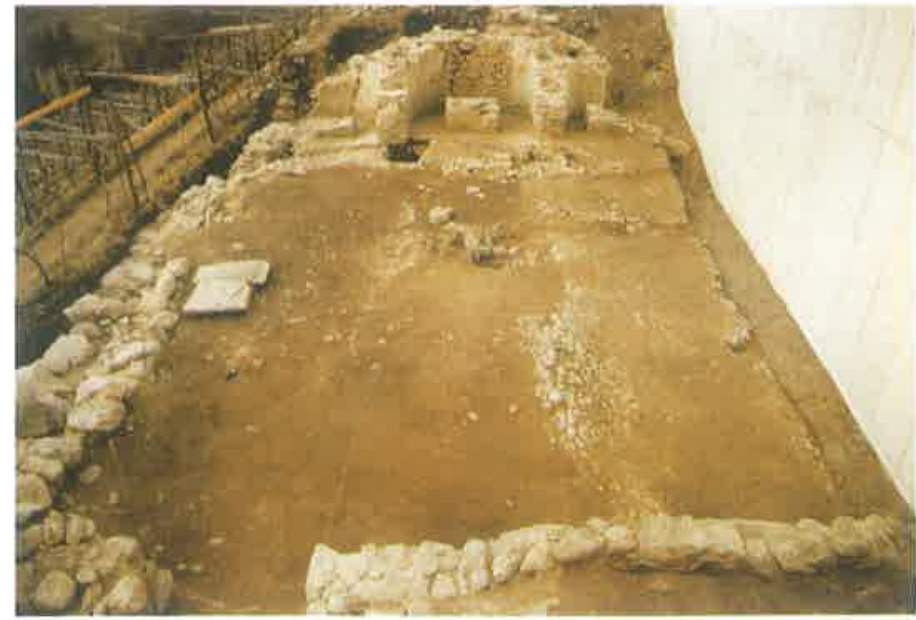

Fig. 3. La chiesa triabsidata nella fase di ricostruzione tarda (da Est). Scavo 1994

generazione prima, dunque verso gli ultimi anni dell'XI secolo?.

Più tardi, ancora su questo lato del colle e parallelamente al muro di navata di monte della chiesa, che in questa occasione fu interamente asportato con le sue fondamenta, fu tracciato un profondo fossato connesso probabilmente con la costruzione dell'antemurale ancora riconoscibile per estesi tratti. Il sistema costituito dall'antemurale e dalla fossa, sarebbe da collocare nella III fase edilizia di Castel Tirolo, protrattasi tra il 1174 ed il $1300^{8}$. Questo complesso di evidenze costituisce ai fini della datazione dell'epilogo di questo edificio sacro un solido terminus ante quem.

Il terreno di livellamento della fase di ricostruzione della chiesa triabsidata sopra descritta, conteneva resti culturali (pietra ollare, ceramica, inoltre una fibbia di cintura di ferro, ossa di animali) ${ }^{9}$.

Datazioni radiocarboniche sono state effettuate su taluni resti di legni carbonizzati che giacevano sul pavimento in calce nell' area del coro, esterna rispetto al riempimento di terra della navata ${ }^{10}$.

Un solido fondamento per una collocazione cronologica della chiesa triabsidata può venire naturalmente, più che dalle scienze ausiliarie dell'archeologia e da considerazioni storiche generali, dal raffronto con edifici simili di queste aree, per i quali una datazione sia sufficientemente accertata.

Il tipo della chiesa triabsidata conobbe una forte diffusione soprattutto nel Canton Grigioni, l'antica Rezia Curiense, nell'VIII e IX sec. ${ }^{11}$. Si è voluto ricercarne l'origine nell' ambito del regno longobardo dove Santa Maria di Aurona a Milano, costruita verso il 740, avrebbe costituito nei decenni successivi un seguito modello ${ }^{12}$. Altri hanno sottolineato la connessione con l'Oriente ed in particolare per quanto riguarda l'Italia Settentrionale il possibile legame con il percorso che era stato secoli prima della via Claudia Augusta, collegante Altino sul margine della laguna dove più tardi sorse Venezia, attraverso la valle dell'Adige con Augusta Vindelicum nella Rezia; di esso in età carolingia conservò importanza assai rilevante il tratto di raccordo che dalla Val Venosta attraverso il varco della Val Monastero raggiungeva Curia (Coira, Chur) ${ }^{13}$. Il particolare tipo dotato di tre absidi non sporgenti verso l'esterno, relativamente raro in questo settore alpino, fu invece assai diffuso ad esempio nella penisola Istriana dove ne sono stati identificati finora non meno di 10 esempi ${ }^{14}$.

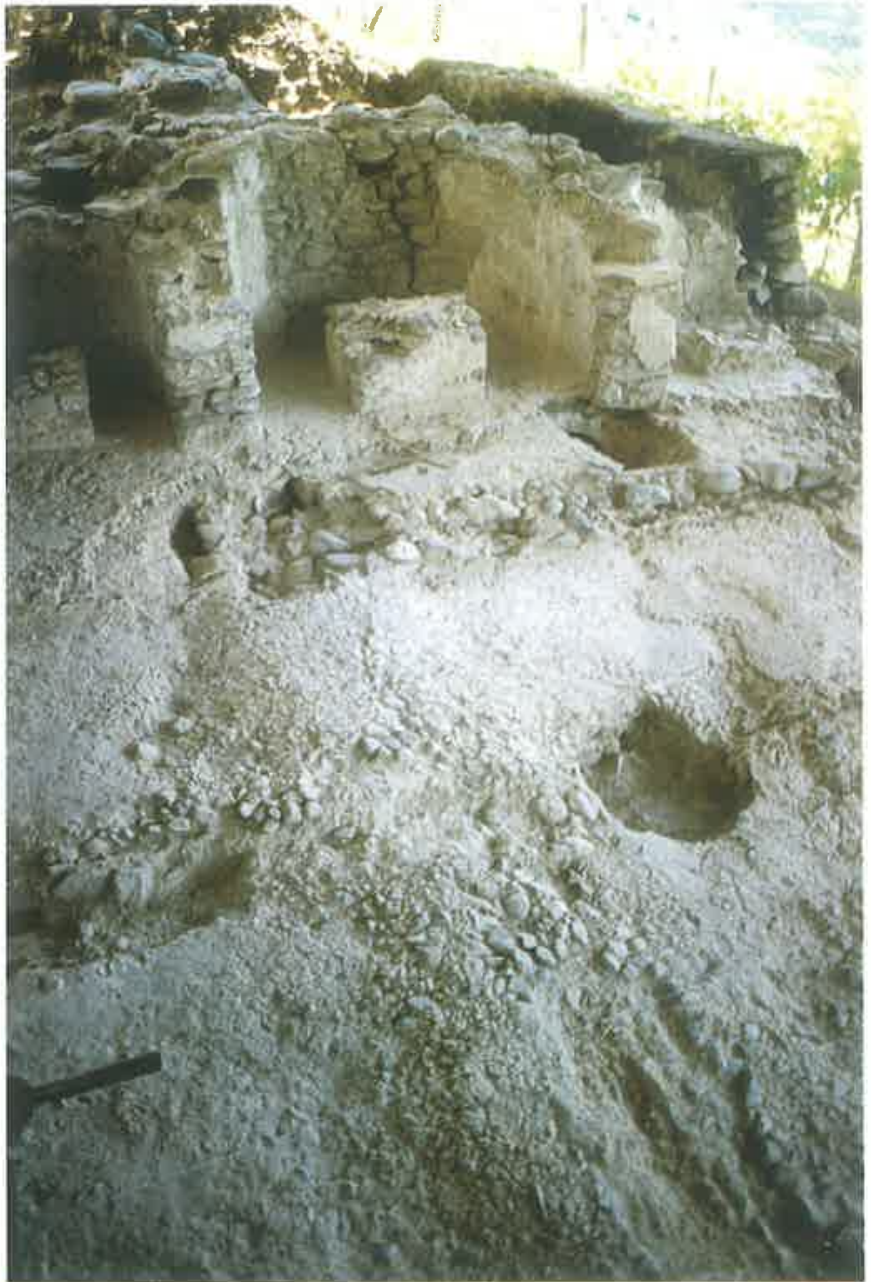

Fig. 4. La chiesa triabsidata. Scavo 1996. È visibile nel pavimento in calce la traccia della base del recinto presbiteriale e al centro la grande buca per palo relativa alle strutture della fase successiva.

In Alto Adige è significativo per noi il caso citato di San Benedetto di Malles in Val Venosta, datato da ultimo verso la metà dell'VIII secolo ${ }^{15}$. L'edificio triabsidato di San Giovanni a Müstair nel Canton Grigioni risale all'incirca all'anno ' $800^{16}$. Sempre sul margine della conca di Merano è stata scavata di recente la chiesa di San Giorgio di Lana, sicuramente a tre absidi (anche se in realtà non è più possibile dire se queste ultime sporgessero all'esterno o fossero inserite nello spessore di un muro esternamente rettilineo come nel caso di Castel Tirolo). A San Giorgio l'esistenza ipotizzata di una precedente chiesa di legno impedirebbe secondo gli illustratori, una datazione anteriore alla prima metà del IX secolo per la successiva fase, quella appunto della chiesa triabsidata ${ }^{17}$. Sempre a Lana sorge la chiesa di Santa Margareta con tre absidi sporgenti verso l'esterno, la quale non è per ora databile con precisione ${ }^{18}$.

Ipotetica è l'attribuzione all'VIII-IX secolo, fatta di recente per la cappella del Castello di Appiano, anch'essa appartenente al tipo triabsidato ${ }^{19}$. Merita di essere citato inoltre il caso di San Bartolomeo di Romeno in cui ad un impianto in origine monoabsidato sono state aggiunte in una seconda fase due absidi laterali ${ }^{20}$.

Decisamente tarde (XI sec.) le grandi chiese rispettivamente del convento benedettino di Castelbadia a Sonnenburg in Val Pusteria e di Santa Maria Assunta a Civezzano presso Trento (in cui alle tre absidi corrisponde ormai un corpo a tre navate $)^{21}$. 


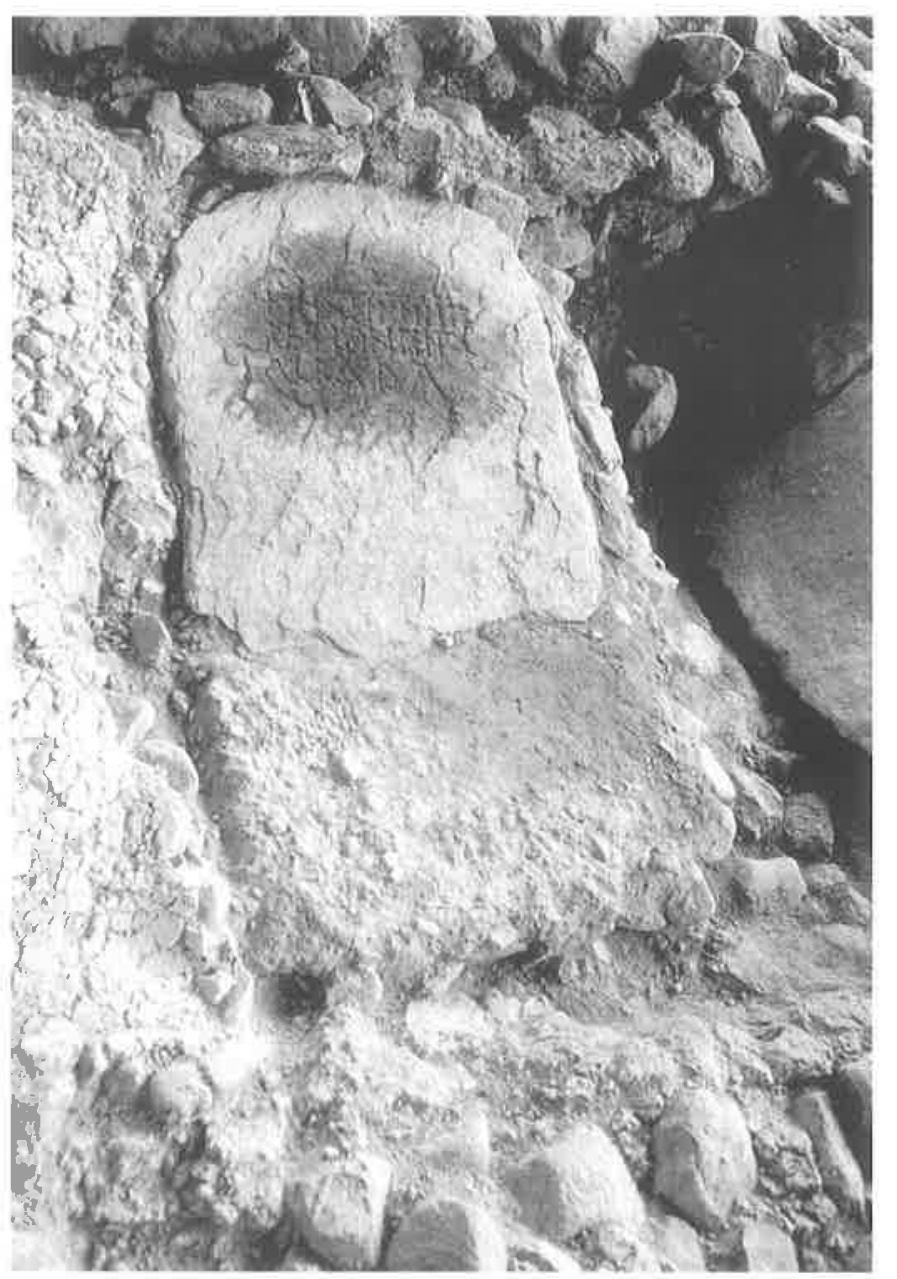

Fig. 5. Lastra tombale in situ con iscrizione riferibile ad un momento avanzato della chiesa monoabsidata; sulla destra lastra di una seconda tomba già violata (scavo 1996).

\section{LA CHIESA PALEOCRISTIANA}

Nel corso dello scavo sono stati a più riprese osservati nella chiesa triabsidata segni di modifiche strutturali che facevano apparire probabile l'esistenza di un precedente edificio sacro. In particolare sulla facciata l'apertura della porta risulta, anziché sull'asse della navata, nettamente asimmetrica e cioè spostata verso valle; il muro occidentale stesso è caratterizzato da un'aggiunta in direzione di monte. Inoltre nel pavimento della navata si nota un impercettibile rialzo che corre da est verso ovest e può corrispondere soltanto alla presenza di un muro sottostante che ha impedito al pavimento di infossarsi come le zone circostanti. Infine sul lato a valle dell'edificio al di sotto dell'abside meridionale compare l'attacco di un corpo murato con andamento ampiamente curvilineo. In una parola sono presenti le tracce di una chiesa di uguale lunghezza, ma di più ridotta larghezza, con grande abside semicircolare, non rientrante rispetto ai muri di navata.

Con certezza poi questo primo edificio fu nella sua fase iniziale costituito da una semplice aula rettangolare mentre l'abside rappresenta un'aggiunta posteriore, secondo un adeguamento architettonico già altre volte evidenziato nell'area norica ${ }^{22}$. In corrispondenza del settore che separa l'abside centrale dall'abside meridionale emergeva da sotto il pavimento della chiesa triabsidata quella che fu giudicata

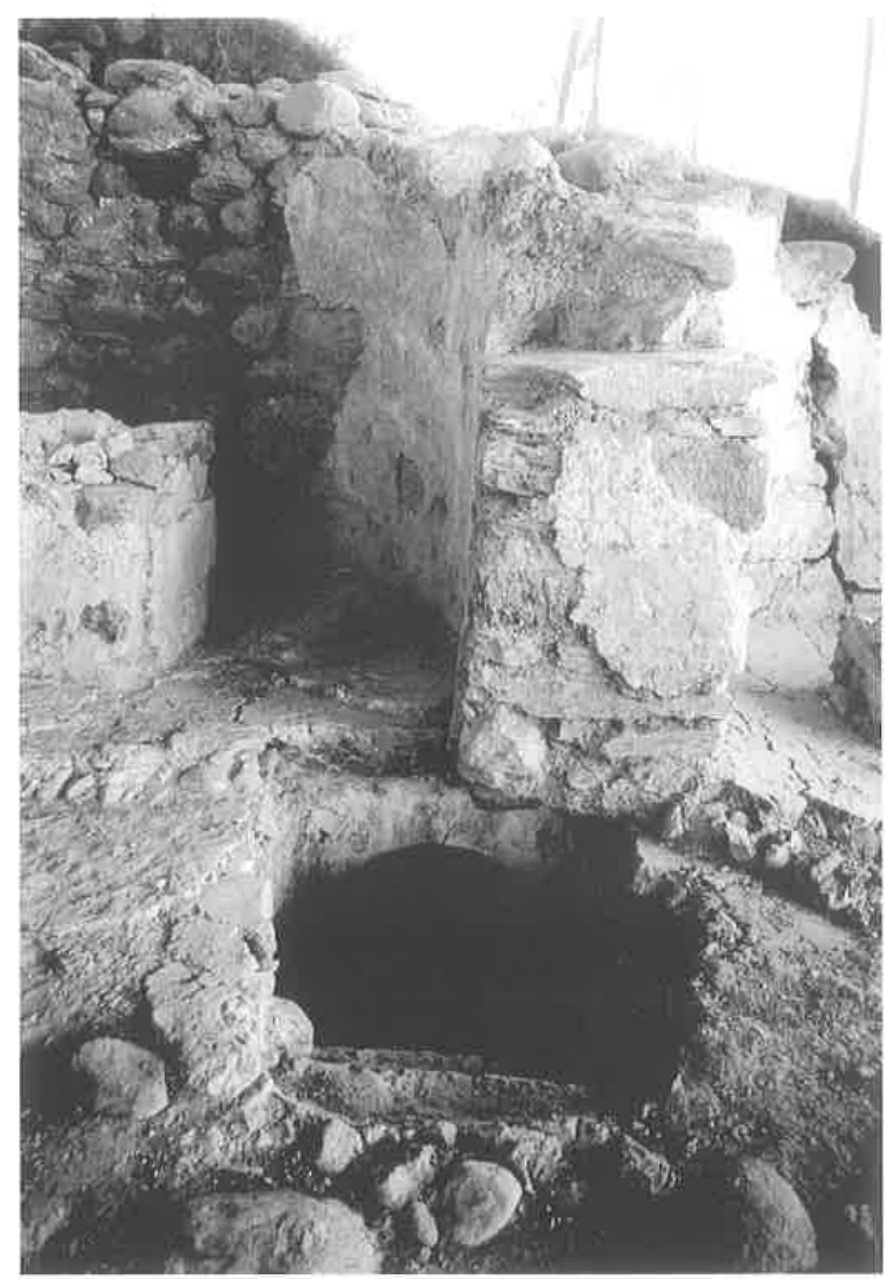

Fig. 7. Il loculo per le reliquie della prima chiesa visibile sotto le murature del setto divisorio tra l'abside centrale e l'absidiola meridionale. inizialmente una lastra tombale parzialmente coperta. Tale lastra, tagliata nella parte mediana, è stata sollevata e asportata mettendo così in luce un regolare riempimento di pietre e malta, la superficie della quale mostrava nettamente impresso un segno a forma di ferro di cavallo ${ }^{23}$. Il riempimento celava a sua volta due gradini disposti a formare una sorta di ripida scala ed inoltre, al di là di una lastra verticale fissata con dei rinzaffi di calce, una nicchia suddivisa in due piani. Nello scomparto superiore era un sarcofago-portareliquie di marmo che conteneva uno scrigno di lamina d'argento con decorazioni dorate a fuoco, all'interno del quale era il complesso delle reliquie avvolte in un minuscolo drappo di seta. Si tratta dunque di un Reliquiengrab collocato sotto l'altare (quest'ultimo asportato completamente) riferibile alla prima chiesa (nella sua fase monoabsidata), secondo il modello aquileiese.

In ogni caso al momento della costruzione della seconda chiesa, quella triabsidata, quando si demolì ed ampliò la chiesa precedente, il sacello fu deliberatamente sigillato, reso inaccessibile e poi probabilmente presto dimenticato con il suo contenuto. Ciò potrebbe essere avvenuto in concomitanza con l'adozione di nuove reliquie giudicate più significative rispetto ad una diversa funzione dell'edificio sacro $^{24}$.

Con certezza appartenevano ancora ad una fase tarda della prima chiesa, cioè quella monoabsidata, una serie di 


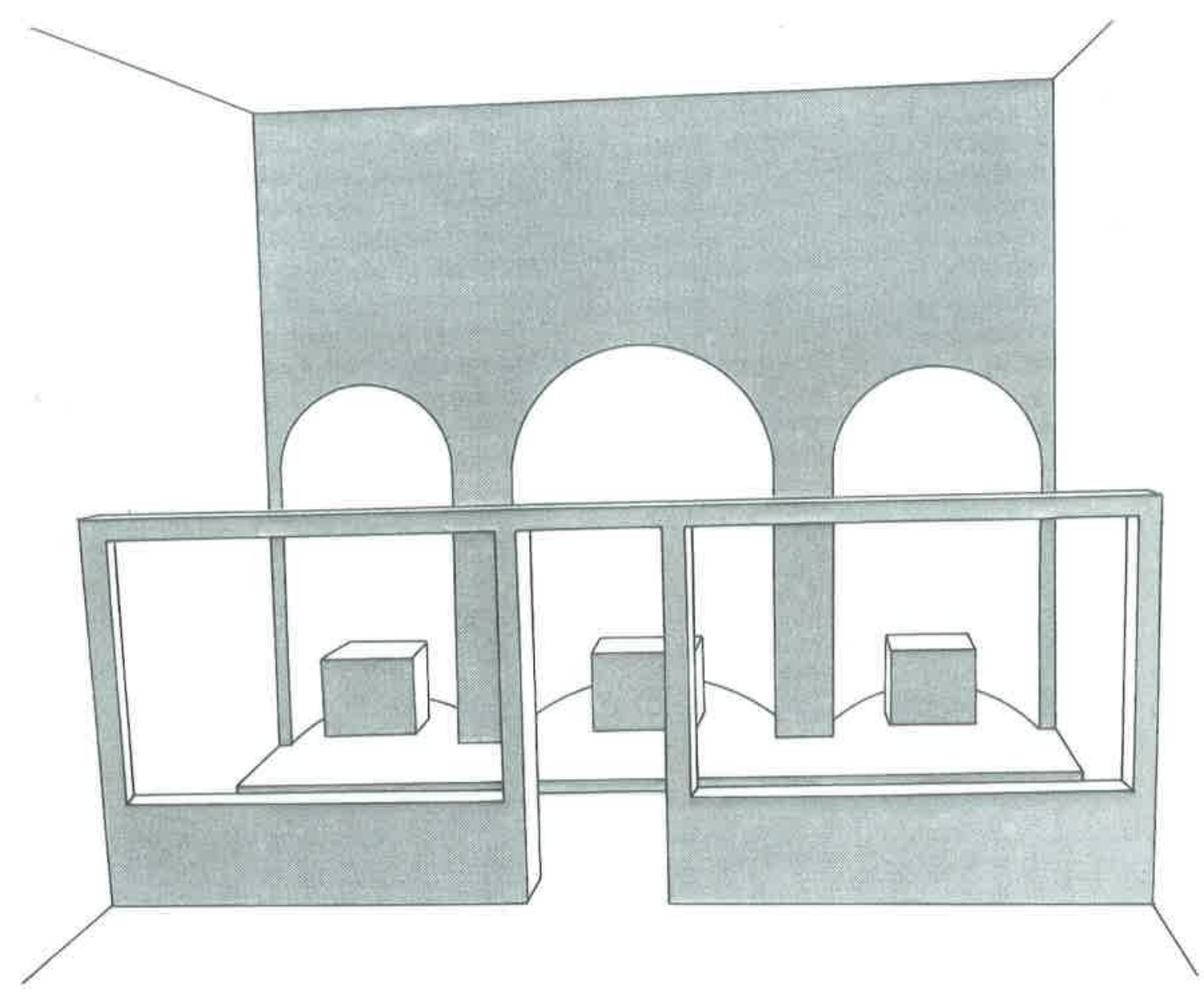

Fig. 6. Ipotesi di ricostruzione della zona del coro della chiesa triabsidata, con recinto presbiterale

sepolture (vedi sopra) collocate sia all'inizio della navata, sia nello spazio tra recinto presbiteriale e altare. In particolare una delle tombe era contraddistinta da una copertura formata da una lastra di scisto rozzamente scalpellata recante un'epigrafe su tre righe. Al di sotto della lastra una struttura tombale apparentemente intatta (una minuscola cista rettangolare di lastre di pietra che riproponeva la forma di una possibile cassetta lignea, al momento dell'apertura in ogni caso dissolta, era del tutto priva di ossa. Ci troviamo probabilmente di fronte ad una situazione di cenotafio $^{25}$. Le altre due tombe erano sconvolte e di fatto private dei resti scheletrici. Ossa umane sono poi venute in luce

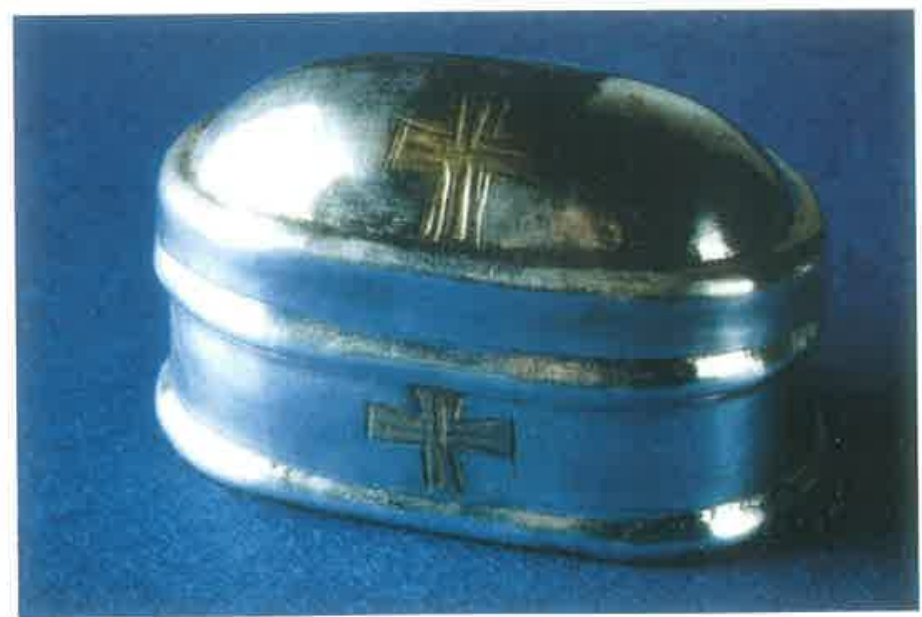

Fig. 8. Reliquiario di lamina d'argento con decorazione dorata a fuoco, rinvenuto nell loculo murato (ricerche 1994). casualmente immediatamente a valle del muro di navata meridonale: un impianto cimiteriale in questo punto e cioè all'esterno appare dunque assai probabile.

Nel 1991 e soprattutto nel 1994 si sono identificati sulla sommità del colle sul lato sud e a sud-ovest rispetto alla chiesa, in più punti tracce di diversi edifici, che soltanto in parte hanno potuto essere esplorati con metodo archeologico Sono state inoltre riconosciute tracce di due sepolture ad inumazione in due punti distinti. I pochi reperti non permettono una datazione precisa, è comunque evidente la realtà di un insediamento (tardo antico-primo medioevale) con edificio per il culto e cimitero, sulla cima del colle dove più tardi fu eretto il castello. La chiesa paleocristiana è interpretabile come la cappella di una comunità vivente nell'ambito di questo castrum di cui non ci è noto il nome antico. Esso costituì più tardi con ogni probabilità uno dei centri satelliti del Castrum Maiense, di cui è conservata menzione in una fonte dell'VIII secolo. Quest'ultimo è da localizzare poco lontano con ogni probabilità nella vasta area che comprende il colle di Zenoburg con il quartiere di Steinach a Merano e Ortenstein ${ }^{26}$.

Vi sono tracce dell'esistenza di un annesso sul lato meridionale a fianco dell'ingresso, sembrano non mancare inoltre tracce di un ulteriore ambiente sul lato orientale, al di sotto del muro dell'abside.

L'edificio sacro presso Castel Tirolo restò dunque in uso per vari secoli assumendo con ogni probabilità nell'VIII se. colo la funzione di chiesa padronale: appare verosimile infatti che avesse stabilito la sua dimora in questa sede un potente locale di origine baiuvara ${ }^{27}$. In un momento ancora successivo, che in ogni caso vorremmo collocare nella se- 
conda metà del X secolo, venne eretta la chiesa triabsidata. Difficile dire che cosa si nasconda dietro a questo evento che comportò tra l'altro l'adesione ad un diverso schema architettonico. Merita peraltro di essere qui almeno riferita la leggenda, secondo cui sulla sommità del colle dove più tardi sorse Castel Tirolo, sarebbe esistito un convento di monache benedettine dipendente dalla Diocesi di Coira; il medesimo sarebbe stato distrutto tra l'altro per far posto al castello. Una contessa di Tirolo (Adelheid, figlia di Alberto III, morta nel 1253) avrebbe fondato più tardi sul fondovalle il Convento di Steinach per riparare a tale atto sacrilego ${ }^{28}$.

\section{ALTRI EDIFICI COEVI DI RECENTE ESPLORATI NEL TRENTINO ALTO ADIGE}

La chiesa di San Pietro nella frazione di Gratsch, di fatto situata a circa $200 \mathrm{~m}$ in linea d'aria dallo scavo descritto di Castel Tirolo e dunque singolarmente vicina, insiste sui resti di una minuscola chiesa paleocristiana con Reliquiengrab, radicalmente demolita; la chiesa attuale viene attribuita dal suo illustratore al periodo tra la metà deI IX e gli inizi del X secolo. Essa testimonia comunque l'adesione ad uno schema architettonico del tutto diverso, con abside mediana esternamente poligonale e con due grandi edicole rettangolari ai lati. Abbondanti resti di stucchi sono attribuibili probabilmente ad un recinto presbiteriale o iconostasi, demolita soltanto nella seconda metà dell'XI secolo ${ }^{29}$.

Nella chiesa di Santo Stefano a Burgusio, situata anch'essa nel Comune di Malles come San Benedetto, sono stati invece condotti scavi nel 1990/91. La chiesa attuale a due piani con un coro quadrato corrisponde già ad una terza fase, ( $\mathrm{X}$ secolo?). Tale edificio insiste infatti sui resti di una chiesa ad aula rettangolare del V-VI sec., sicuramente modificata nel VII con l'aggiunta di un annesso sul lato meridionale ${ }^{30}$.

\section{SCULTURE DEL IX-X SECOLO NEL TERRITORIO TRENTINO- ALTOATESINO TRA VIII E X SECOLO}

Problema aperto è l'esistenza nella chiesa triabsidata di Castel Tirolo di un arredo di marmi architettonici. Nulla è stato rinvenuto in scavo, eppure la struttura di un recinto presbiteriale era presente, perché ne è stata riconosciuta la base di malta.

Appare in ogni caso interessante problema filologico ricercare se alcuni almeno degli elementi riconosciuti di recente come spoglie reimpiegate, nella cappella romanica del castello ${ }^{31}$ provengano in realtà da questa chiesa. Ci riferiamo in particolare ad una serie di pilastrini verticali con funzione di stipiti nella strombatura del portale della cappella. Quello di sinistra reca un motivo vegetale (si tratta di una palma stilizzata) sulla parte frontale e un motivo a grandi anelli intrecciati sulla superficie laterale ${ }^{32}$, sicuramente affine come schema almeno, a quello che compare su due pilastrini angolari di recinto presbiteriale da Santa Maria Maggiore a Trento ${ }^{33}$. Altri esempi sono noti da Cividale $^{34}$. Potrebbe dunque trattarsi nel nostro caso di uno dei pilastrini di un recinto presbiteriale, le cui dimensioni (altezza cm 110, larghezza cm 30) si ripetono esattamente sullo stipite del lato opposto dove è presente un diverso motivo (tralci con foglie di vite e grappoli). La tecnica del rilievo, un certo realismo descrittivo nella resa della pianta (la conformazione ad es. della foglia di edera nella parte inferiore), suggeriscono una datazione molto avanzata (ultimi decenni del X secolo-primi dell'XI?) nell'ambito della scultura tardo-carolingia ottoniana. Quest'impressione è accresciuta dalla trattazione della treccia a volute particolarmente ampie e tondeggianti sulla faccia interna che ricorda da vicino un capitello a stampelle conservato a Trento (forse proveniente dal palazzo del vescovo Udalrico, prima metà del sec. XI) e altre spoglie come ad esempio un fregio con grappoli e foglie di vite, provenienti dal duomo della medesima città; anche un capitello con decorazione a treccia da Mezzocorona sembra mostrare qualche analogia ${ }^{35}$.

Stilisticamente questi marmi hanno affinità ormai soltanto remote con il complesso delle sculture propriamente carolinge finora note dall'Alto Adige occidentale, cioè dalla Val Venosta (da Malles-Mals, Lasa-Laas, Corces-Kortsch, Glorenza-Glurns e ultimamente da Covelano-Göflan) ${ }^{36}$

Si differenziano anche dalle importanti presenze di scultura carolingia note nel vicino territorio del Trentino occidentale, cioè dalla Valle del fiume Sarca. Sono qui venute in luce sculture ad intreccio nell'alta valle a Spiazzo presso Tione (San Vigilio) e a Tione stesso (Santa Maria); nella media valle in corrispondenza dell'altipiano del Bleggio, a Lundo (San Marcello), a Santa Croce (chiesa dei Santi Dionisio, Rustico ed Eleuterio), a Vigo Lomaso (San Lorenzo), a Seo, Poia e Premione, inoltre al castello di Stenico (San Martino); infine nella piana di Riva dove il fiume sbocca nel lago di Garda, a Vignole (San Valentino), a Varignano e a Bolognano (chiesa di Santa Maria) presso Arco; a Varone (Santa Maria); a Tenno (San Lorenzo); infine a Riva del Garda stessa (San Cassiano, inoltre in altri edifici sacri non meglio precisati) ${ }^{37}$, al castello di Drena (chiesa di San Martino) ${ }^{38}$. Questa presenza ebbe da un lato (verso Occidente) stretti, quanto ovvi contatti con il centro artistico di Brescia di cui deve considerarsi una emanazione, dall'altro fece sentire a sua volta palesi influssi verso nord est fino alla città di Trento, l'antico Municipium Tridentum, (chiesa dei Santi Cosma e Damiano sul Dos Trento, Santa Maria Maggiore e rispettivamente San Vigilio, l'attuale duomo) ${ }^{39}$ inoltre verso nord, cioè verso la valle di Non, a Sanzeno (chiesa dei Santi Sisinio, Martirio e Alessandro). Nella Val d'Adige a sud di Trento altri esempi sono noti da Chizzola (Santa Cecilia) e da Avio; inoltre da Mori, Brentonico e Castione ${ }^{40}$. Un esempio isolato, verosimilmente tardo, è noto inoltre $a$ Mezzocorona alla confluenza della Val di Non nella Val d'Adige $^{41}$

Per ora isolati e pertanto difficilmente inquadrabili due esempi di sculture a intreccio da Sabiona in Val d'Isarco (chiesa di San Cassiano), affluente dell'Adige e pochi frammenti di capitello da San Candido in Val Pusteria già nel bacino della Drava, affluente del Danubio ${ }^{42}$.

Si è cercato di eviderfziare in passato la coincidenza significativa per le sculture del bacino del Sarca con la realtà di cave di arenaria ad Arco, e rispettivamente di cave a Lundo, invece per le sculture venostane con l'esistenza di affioramenti di marmo microcristallino principalmente a LasaLaas e a Covelano-Göflan ${ }^{43}$.

Se ne ricava che in tutte le aree circostanti dove esempi di sculture architettoniche di questo genere finora non sono attestati, e dove edifici sacri di questo periodo si possono ipotizzare in numero non meno grande che altrove, potrebbe essere in realtà ragionevole pensare ad una forte produzione di legni intagliati, ora per ovvi motivi completamente scomparsi, che non necessariamente dovettero rientrare nel novero di un'arte povera o minore.

E' tuttavia degna di attenzione l'opinione di recente espressa che la diffusione di sculture architettoniche a treccia 

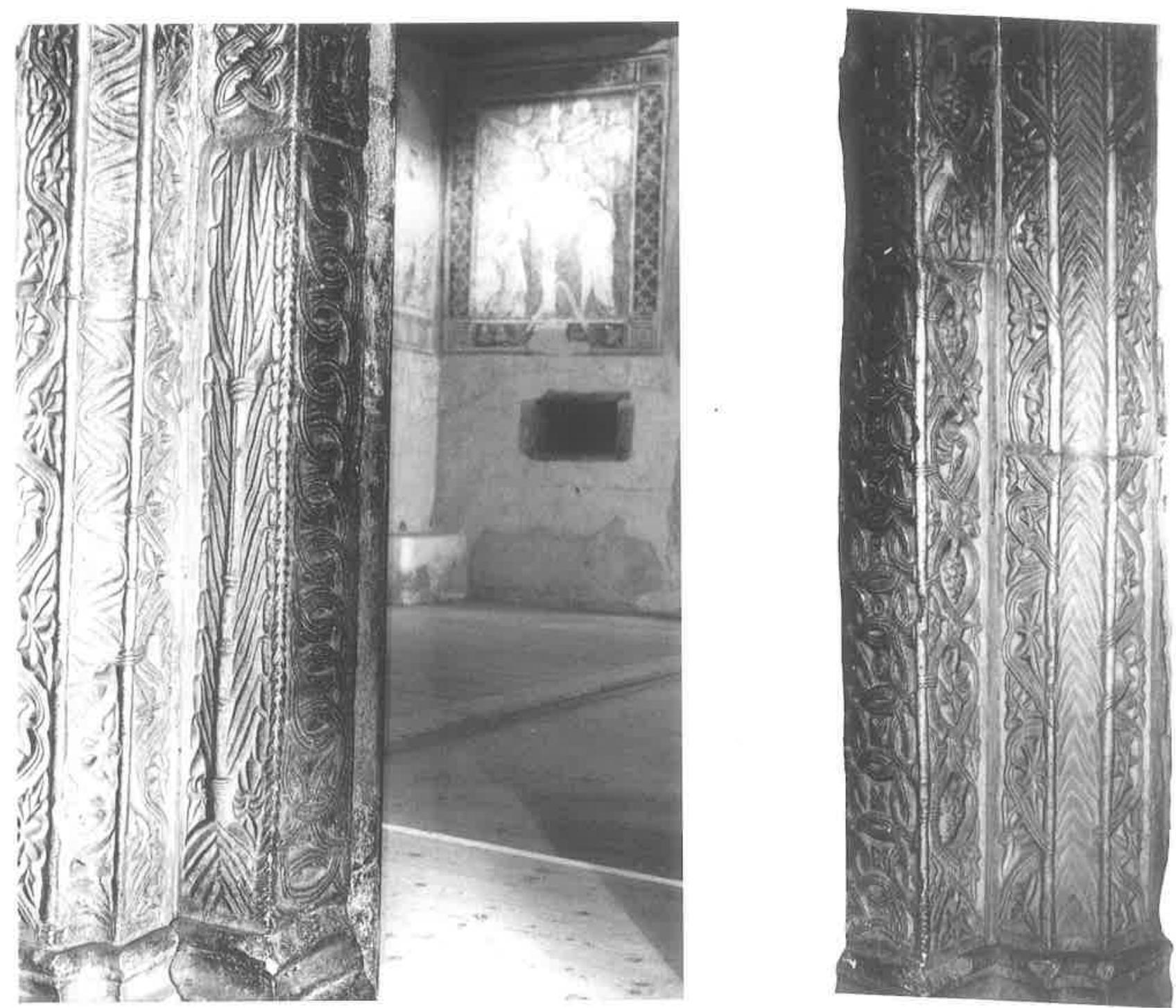

A
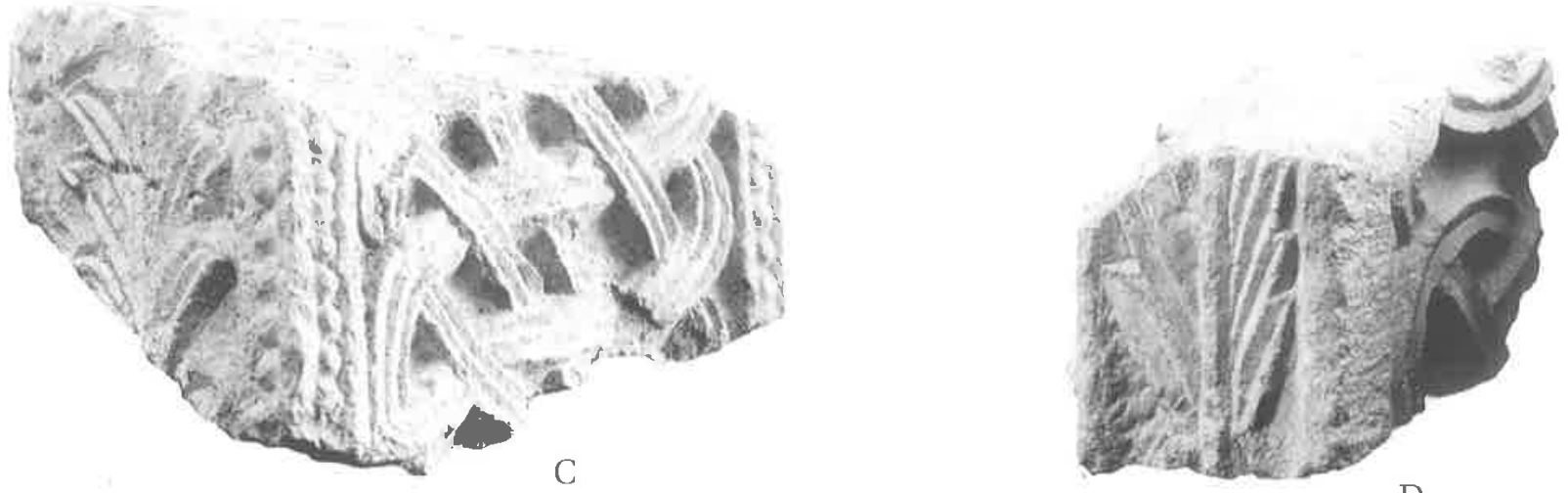

$\mathrm{D}$

T. 1. Castel Tirolo presso Merano. Portale composito della cappella: A) stipite interno sul lato settentrionale con il motivo della palma e con motivi a treccia sul lato interno; B) stipite di destra con motivo a grappolo d'uva e intreccio sul lato interno; C) D) frammenti di pilastrino dagli scavi di Santa Maria Maggiore a Trento (arch. Uff. Beni Archeologici, Trento) 

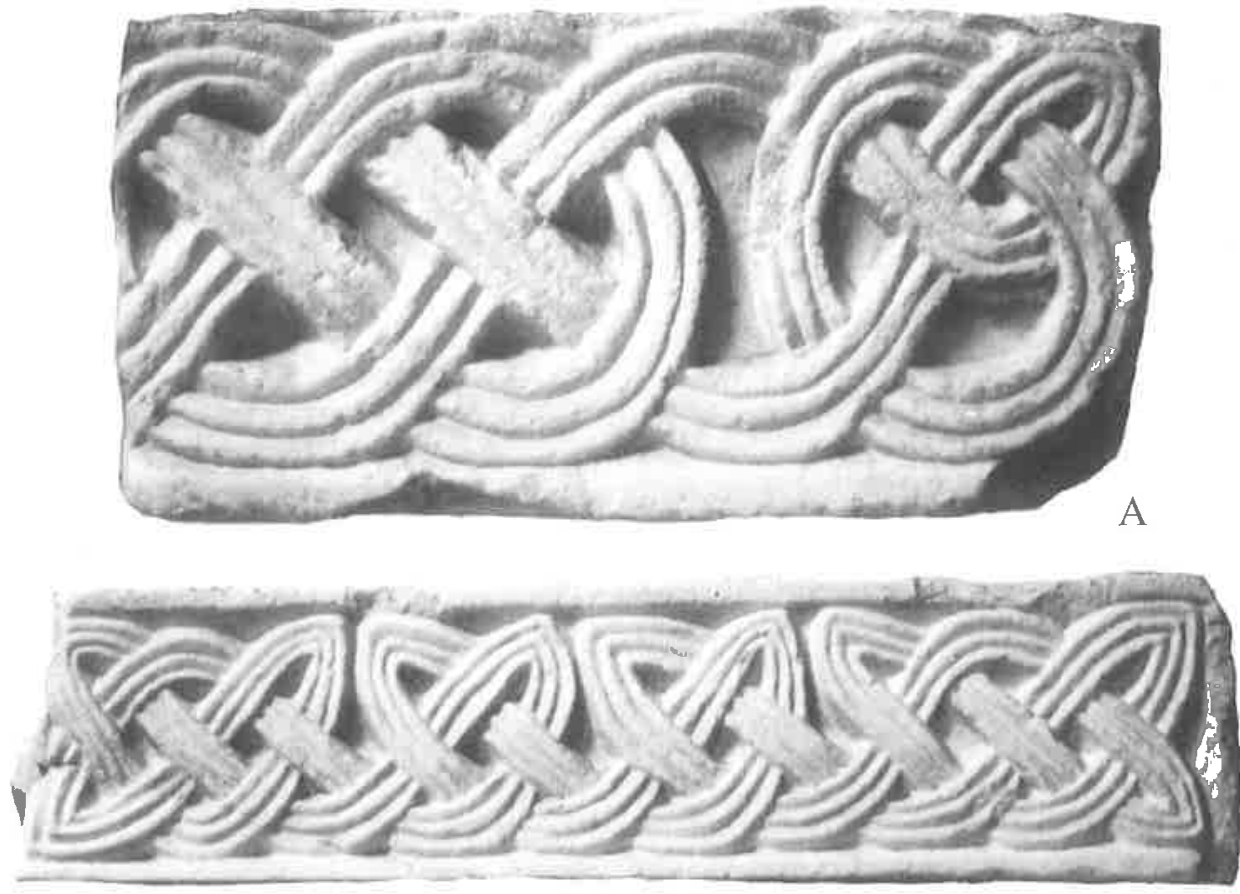

B
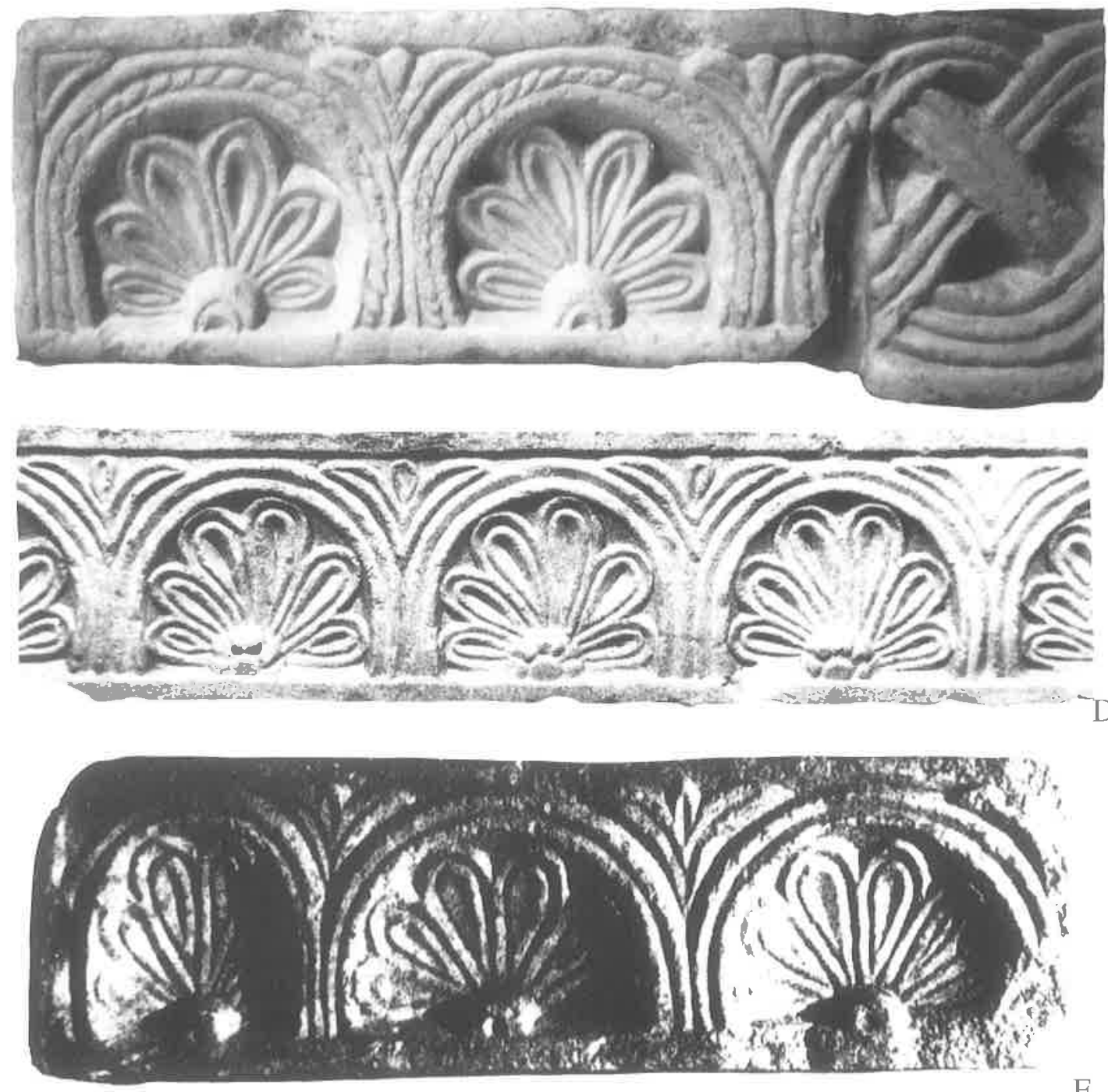


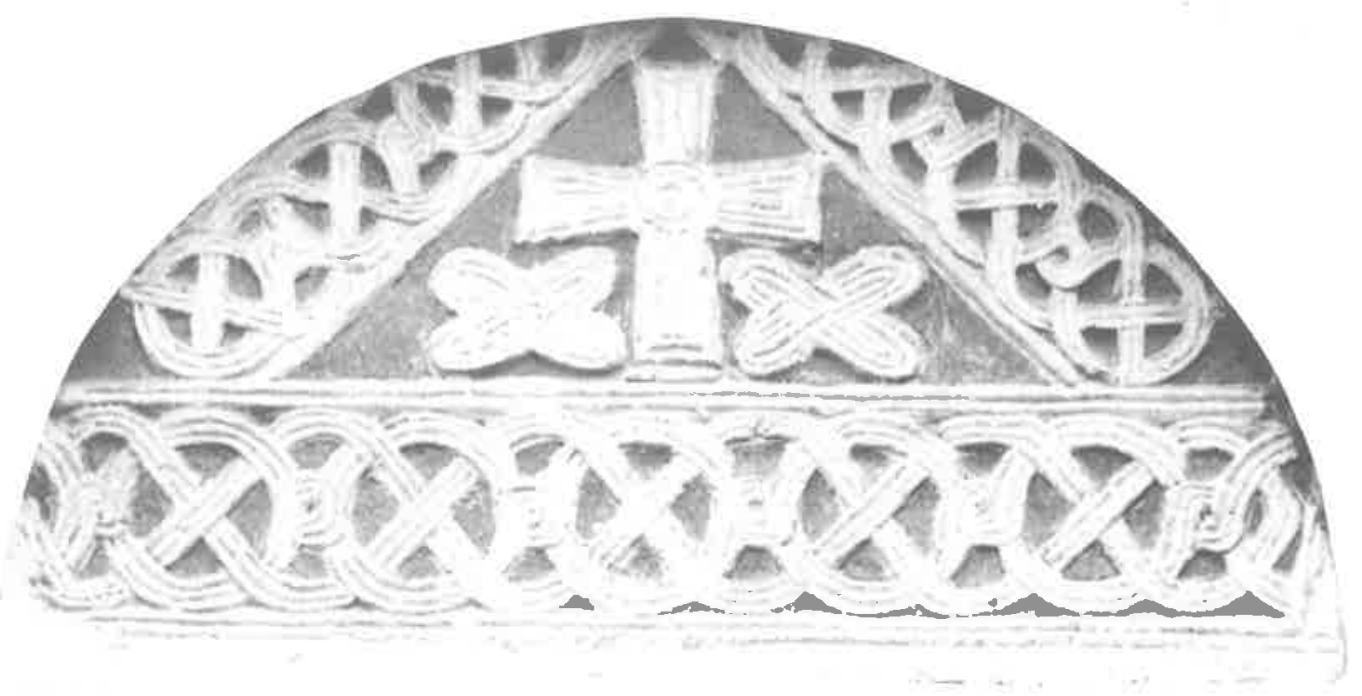

A

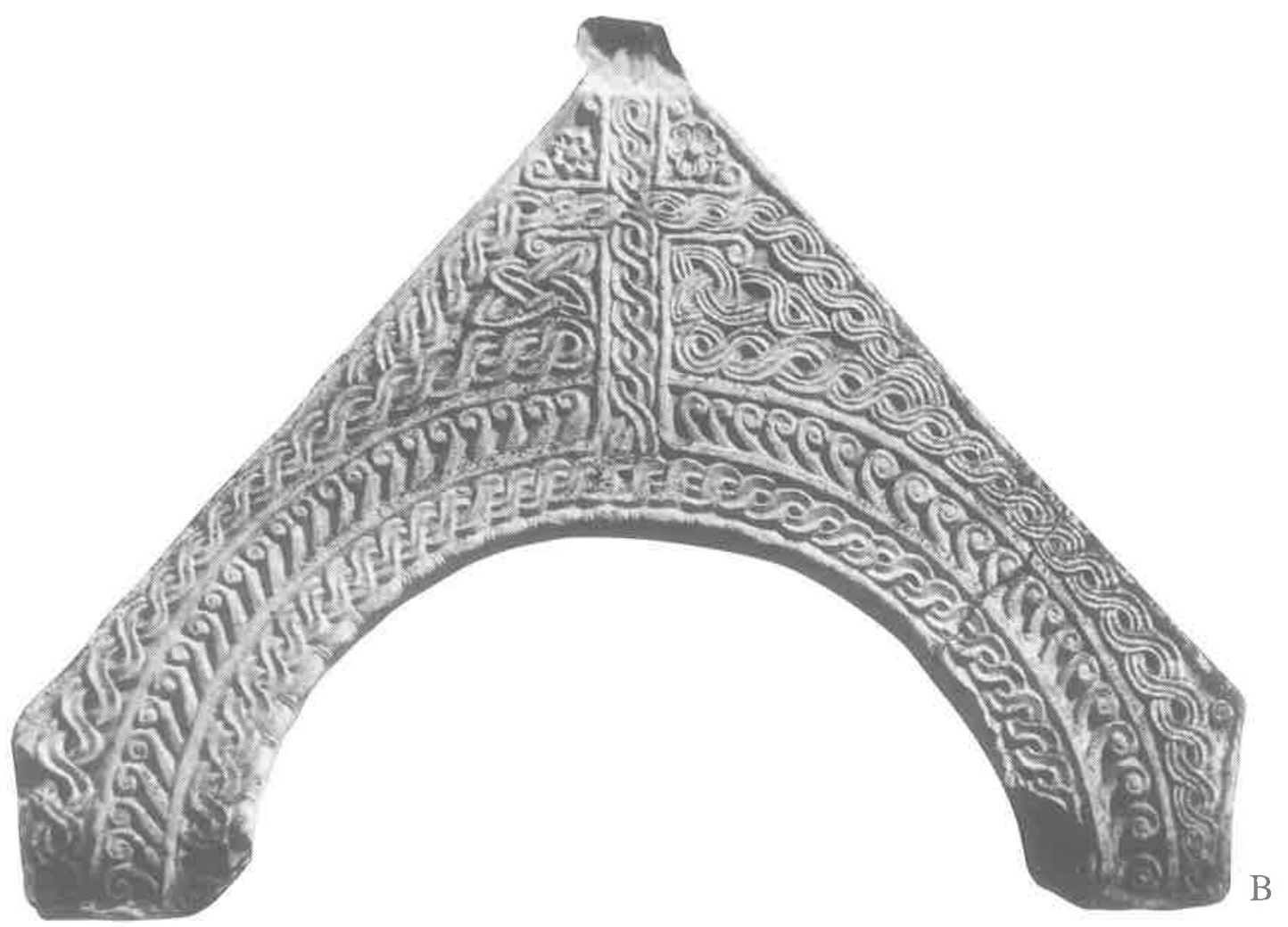



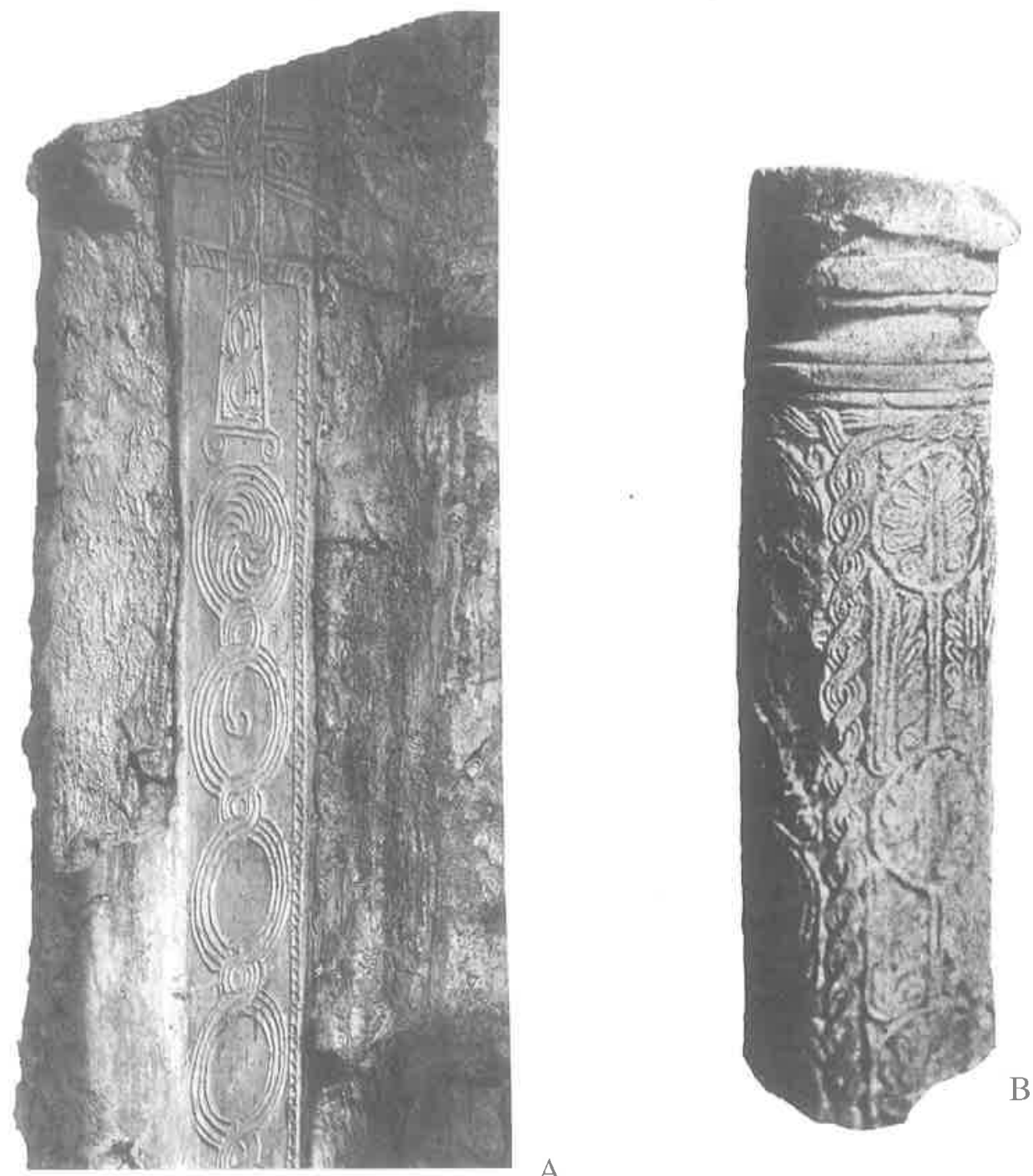

A

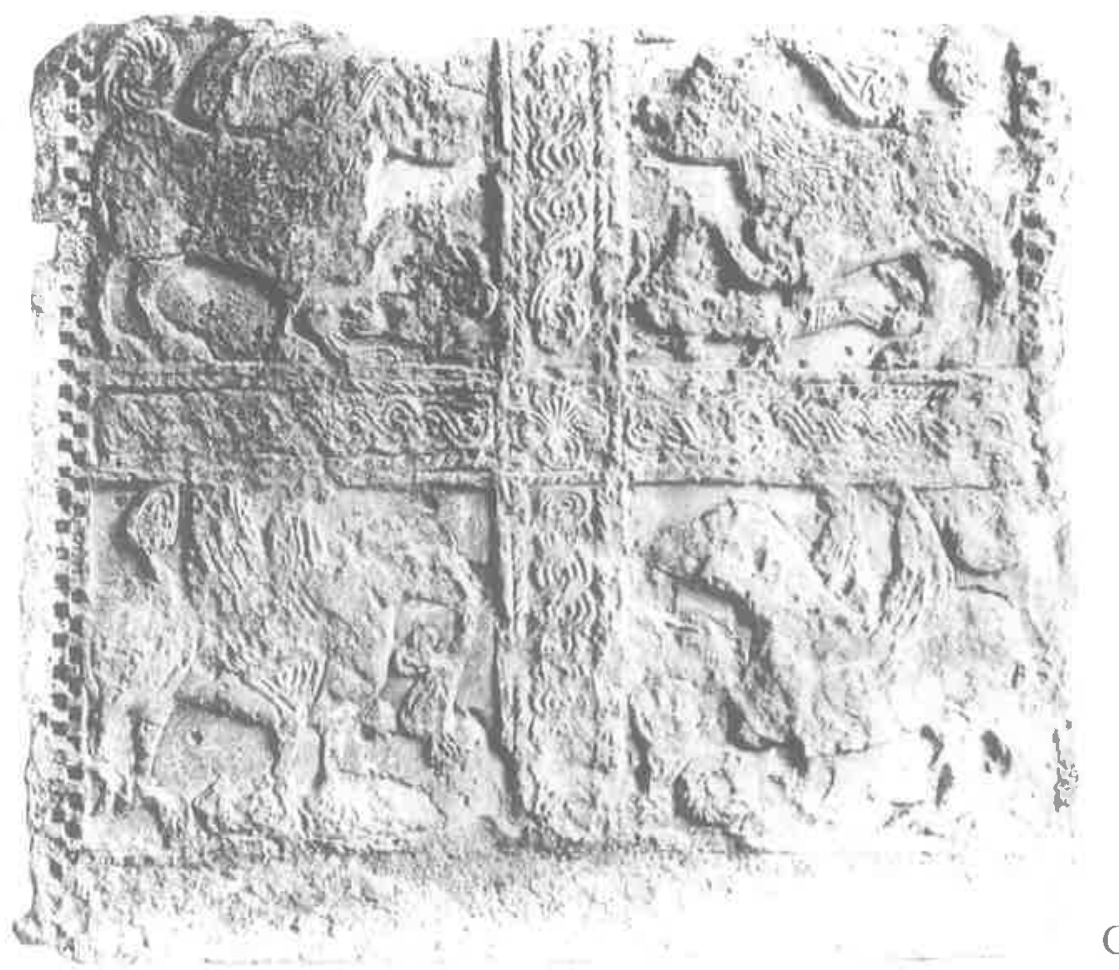



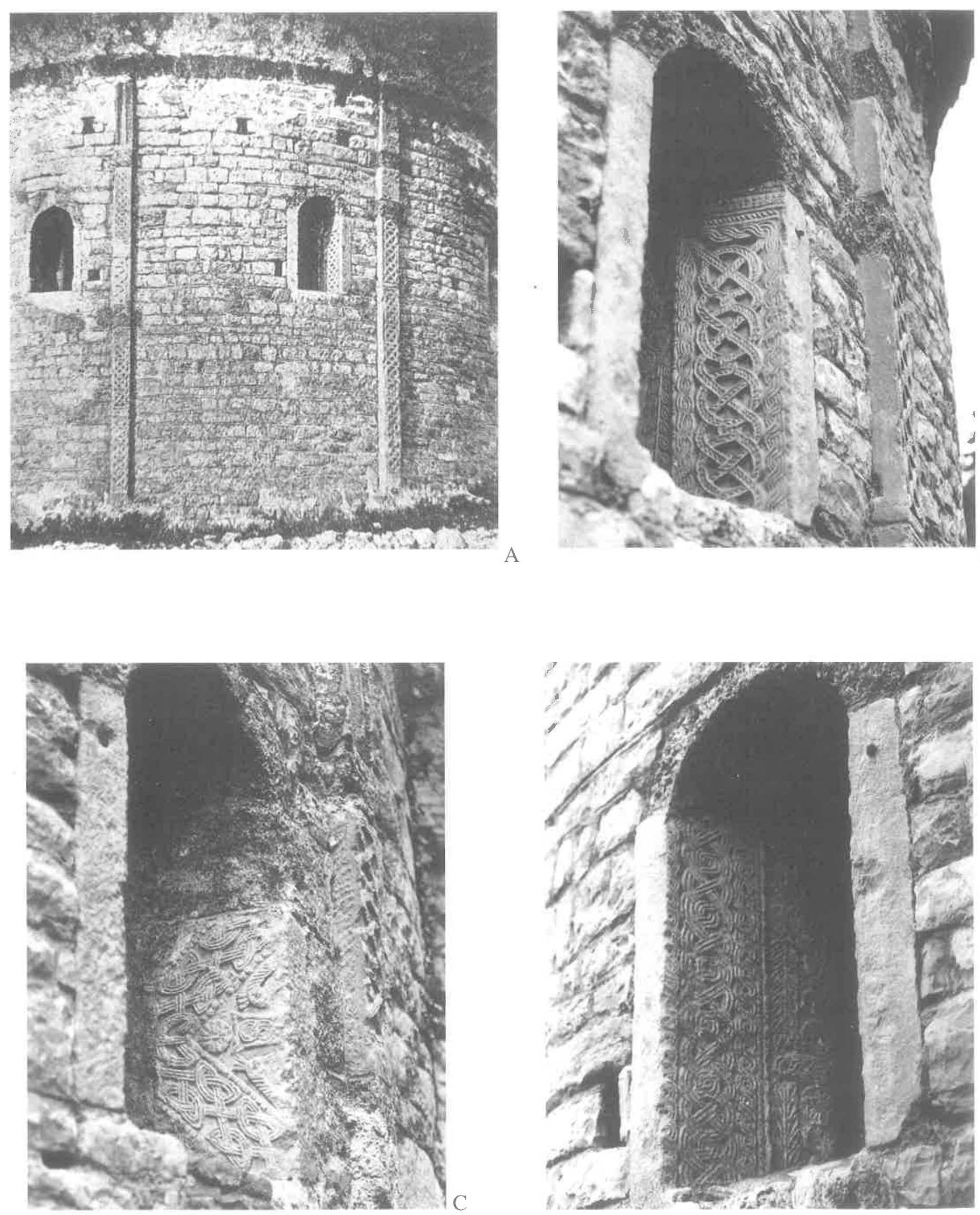

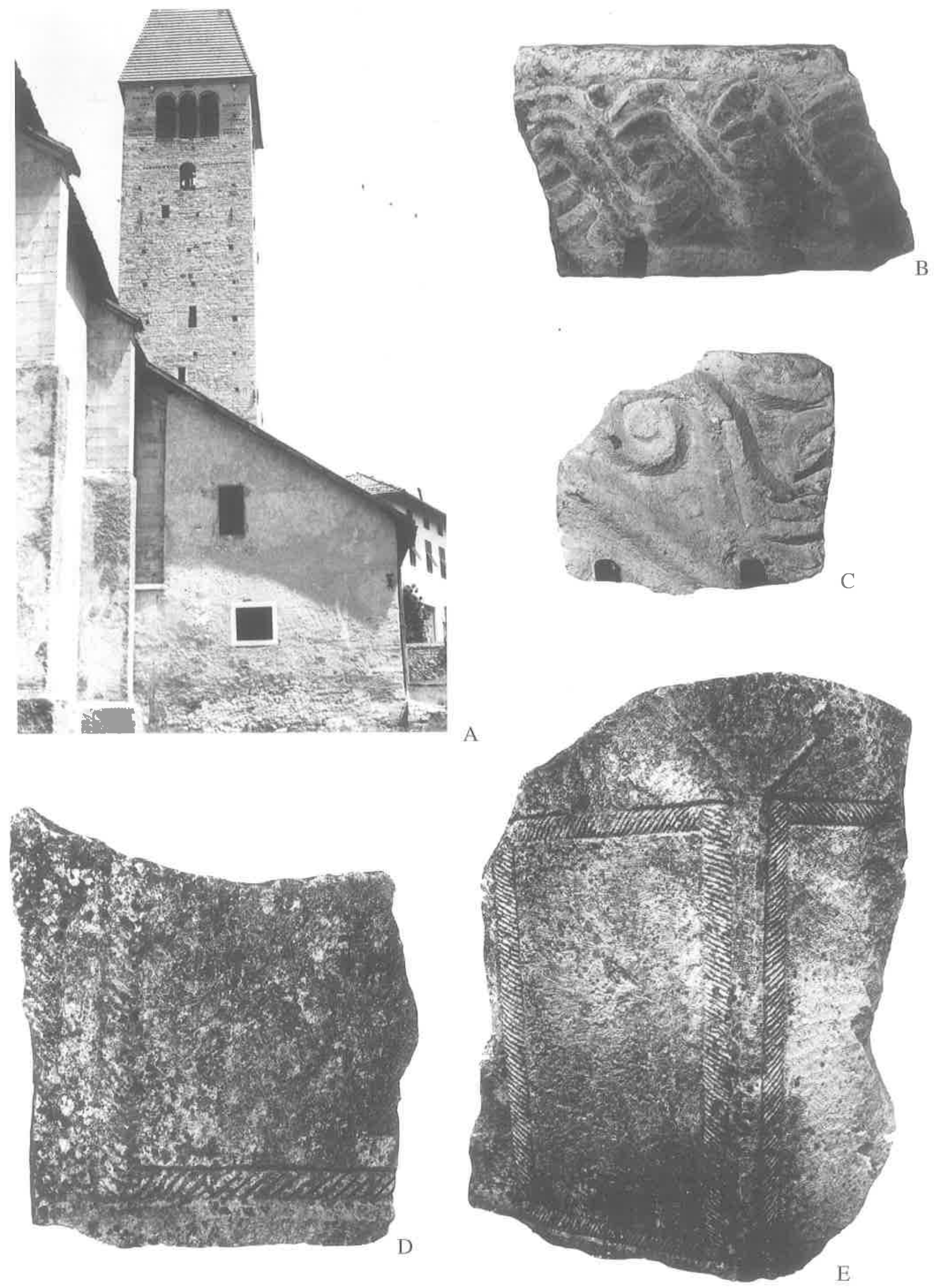

T. 6. Sanzeno, Val di Non (Trento). Chiesa dei Santi Sisinio, Martirio ed Alessandro: A) veduta della chiesa da Sud, B) frammento di cornice con motivo a treccia, calcare ammonitico rosso, larghezza $\mathrm{cm} \mathrm{15,} \mathrm{C)} \mathrm{frammento} \mathrm{con} \mathrm{motivo} \mathrm{a} \mathrm{caulicoli,} \mathrm{D)} \mathrm{e} \mathrm{E)} \mathrm{due} \mathrm{frammenti} \mathrm{di} \mathrm{un}$ probabile ambone in arenaria (altezza superstite $\mathrm{cm} 71 \mathrm{e} \mathrm{cm} \mathrm{84),} \mathrm{sul} \mathrm{lato} \mathrm{esterno} \mathrm{della} \mathrm{sacrestia;} \mathrm{B)} \mathrm{e} \mathrm{C)} \mathrm{ora} \mathrm{nel} \mathrm{giardino} \mathrm{dell'ex-canonica,}$ adiacente alla chiesa. 

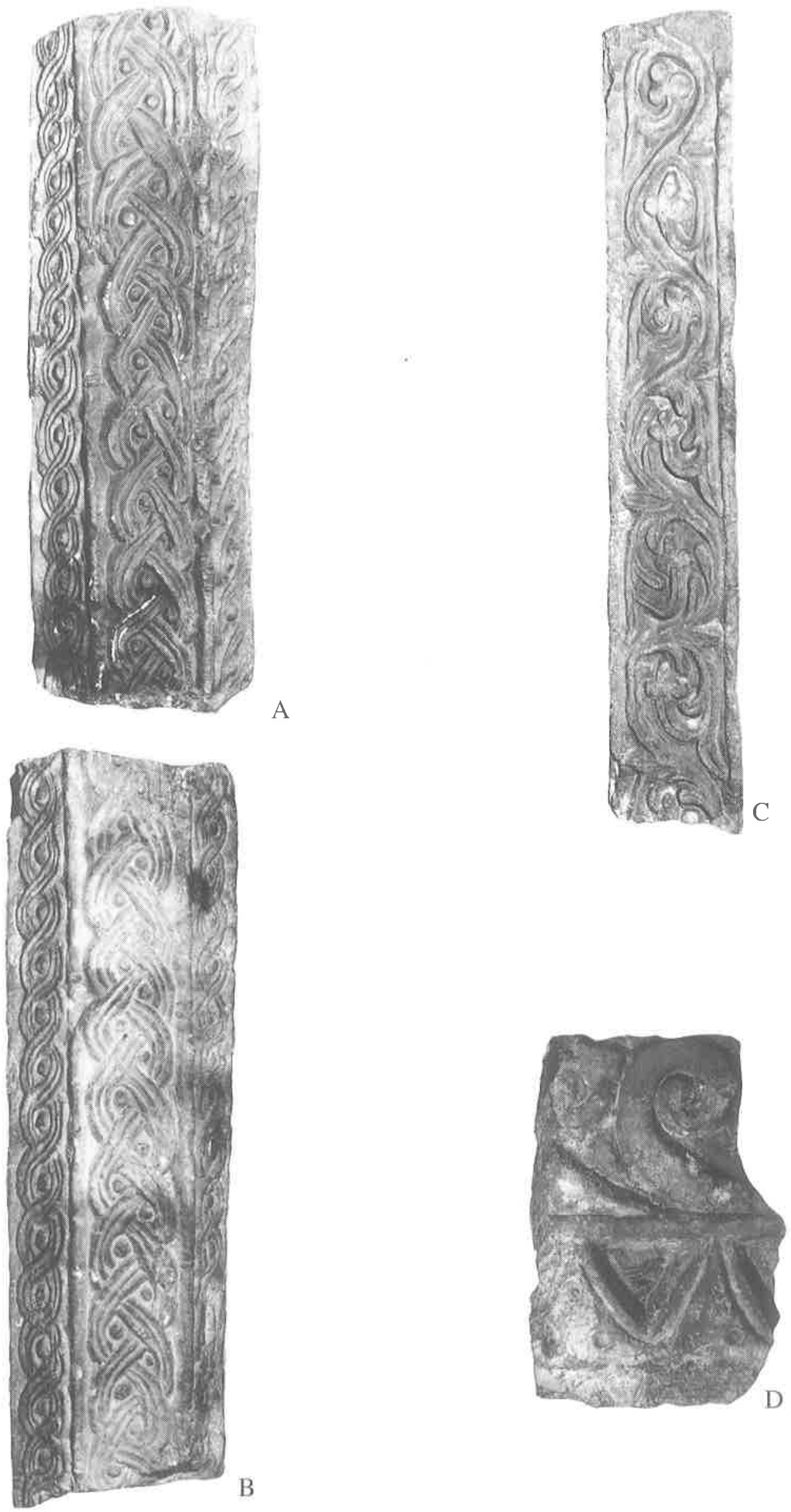

T. 7. Sanzeno, Val di Non (Trento), Chiesa dei Santi Sisinio, Martirio ed Alessandro: A) e B) frammenti di un medesimo pilastrino ottagonale già murati nel campanile, altezza cm 60 e cm 54, C) altra faccia del pilastrino, D) frammento con motivo a caulicoli, ora all'interno della sacrestia vecchia. 
di epoca carolingia, soprattutto in aree marginali rispetto all'ambiente di formazione primario e cioè l'Italia centrale e settentrionale, debba essere messa in relazione con fatti storici precisi, considerati su base strettamente regionale ${ }^{44}$.

Per il bacino del Sarca indubbiamente d'importanza inusuale il citato ricco complesso dell'abside di San Lorenzo a Tenno, dove sono murati 19 frammenti, in parte coerenti fra loro, costituiti da parti di plutei, pilastrini e architravi di recinti presbiteriali e cibori. Vi sono forti differenze di stile da oggetto ad oggetto, inoltre si osserva una palese ripetizione di manufatti con funzioni analoghe, per cui l'ipotesi di una raccolta di sculture da edifici sacri diversi, esistenti nei dintorni, appare senz' altro probabile ${ }^{45}$.

Di speciale interesse inoltre sono poi i ricchi complessi citati di Vigo Lomaso e di Santa Croce nel Bleggio e soprattutto quello del Castello di Stenico ${ }^{46}$.

Dopo le anticipazioni di Orsi e di Atz e gli studi di Cecchelli e di Rasmo, un riesame sistematico della produzione regionale di epoca carolingia appare importante ${ }^{47}$.

In particolare poi pressoché inedito è il complesso di Sanzeno in Val di Non ${ }^{48}$, rinvenuto nell'ambito dell'antichissima chiesa dedicata ai Santi missionari Sisinio, Martirio e Alessandro, che subirono il martirio, forse nel luogo stesso dove oggi sorge la chiesa, il 29 maggio del 397 d.C.. Sono presenti due frammenti di (un medesimo?) pilastrino ottagonale decorato ${ }^{49}$, un frammento di architrave di recin- to presbiteriale, un frammento di timpano con caulicoli, tutti riferibili ad un recinto presbiteriale. Un frammento di cornice di recinto presbiteriale in calcare ammonitico rosso ed un frammento di timpano con caulicoli conservati in un edificio vicino (ex casa canonica), provengono anch'essi con ogni probabilità dal sottosuolo della chiesa. Infine due frammenti in forma di vasca, ora addossati al muro dell'attuale sacrestia, sembrano potersi ricondurre ad un ambone di semplice fattura, decorato unicamente da fuserole a cordicella, disposte a mo' di lesene e collegate tra loro superiormente da timpani. Raffronti con possibili prototipi bizantino-ravennati come ad esempio l'ambone (peraltro alquanto più antico) della basilica ariana di Ravenna dove è presente il motivo della "fronte di palazzo" con colonne tortili collegate da due archi ai lati e da un timpano al centro, appaiono abbastanza convincenti ${ }^{50}$. Gli specchi delimitati da queste cornici a fuserola sono del tutto privi di decorazioni (incompiuti?). Si tratterebbe di uno dei rari esempi di ambone nell'alto bacino dell'Adige e territori limitrofi (assieme all'esemplare, quest'ultimo invece riccamente decorato, da Santa Croce nel Bleggio, di recente recupero) $)^{51}$. Il tutto attesta un'importante ristrutturazione riferibile all'VIII-IX sec., della primitiva chiesa paleocristiana, i resti della quale si nascondono al di sotto del pavimento della cappella detta dei Martiri, la quale si dispone oggi perpendicolarmente alla navata della chiesa attuale, costruita nel $1472^{52}$.

${ }^{1}$ Cfr. la pubblicazione-catalogo: Rasmo et alii, 1981.

${ }^{2}$ Cfr. ORSI 1883; GARBER 1915; GEROLA 1926; CECCHELLI 1928; EGG 1973; RASMO 1976. Meritano tuttavia menzione le ricerche di Rasmo (1946-47), in occasione della ricostruzione del Duomo di Bolzano, distrutto dai bombardamenti, nel corso delle quali fu individuata una fase edilizia carolingia entro il perimetro della grande basilica paleocristiana. A tale fase furono in ogni caso attribuiti alcuni frammenti di affresco rinvenuti nei riempimenti accanto al muro sud della navata. Qualche anno più tardi nella città di Trento, I. Rogger condusse ampie indagini dentro il Duomo (cfr. ROGGER, 1967, $68,73,74)$. Nella medesima città, presso la chiesa di Santa Maria Maggiore fu tra l'altro portato in luce negli anni settanta un ricco complesso di marmi architettonici relativi a quest'epoca (cfr. BOSCHI CIURLETTI, 1978). Per gli scavi di San Pietro di Quarazze/Gratsch cfr. LUNZ, 1978. Per gli scavi di Sabiona-Säben cfr. BIERBRAUER--NOTHDURFTER, 1986.

${ }^{4}$ Il ritrovamento è stato segnalato all'Ufficio Beni Archeologici della Soprintendenza Prov. ai Beni Culturali dalla dott.ssa Catrin Marzoli, allora ispettrice del Museo Provinciale di Archeologia a Castel Tirolo. La medesima ha collaborato con l'autore in tutte le fasi successive delle ricerche, assieme a Gino Bombonato della cooperativa Scavi e Restauri di Bolzano. Ambedue hanno inoltre prestato concretamente il loro aiuto nella composizione di queste righe.

${ }^{5} \mathrm{Nel} 1991$ venne recuperto nel terreno superficiale nei pressi della chiesa uno spillone in bronzo a gambo decorato, attribuibile al VI-VII sec. (segnalazione Stefan Demetz). Dal colle del castello era nota nel XVII sec. una statuetta bronzea di Iside (... repertum est hoc in pago hodierno Tirol ad arcem principem unde toti regioni nomen est olim teriolis dictus ...) (ROSCHMAN 1756, capitolo "Teriolis et tractus meranensis cum Valle Venusta").

${ }^{6}$ Sul tema delle chiese ad aula unica triabsidate cfr. in particolare le pubblicazioni OSWALD, SCHÄFER, SENNHAUSER, 1966, inoltre SENNHAUSER 1979). Appunto in considerazione di questa competenza specifica fin dall'inizio di queste ricerche abbiamo ricercato la consulenza del Prof. Rudolf Sennhauser dell’Università di Zurigo, direttore degli scavi di San Giovanni di Müstair nella vicina Val Monastero.

${ }^{7}$ Cfr. NICOLUSSI, 1995.

${ }^{8}$ Cfr. ERHARD, 1995, pp. 64-67 (inoltre cfr. scheda di M. BITSCHNAU e W. HAUSER, pp. 72-73).

${ }^{9} \mathrm{E}^{\prime}$ stato possibile negli anni scorsi pubblicare un complesso di ceramiche provenienti dallo scavo dei resti di un edificio a Bolzano, (piazza Walther, 1984) (cfr. DAL RI, 1991, p. 254). La costruzione di tale edificio (che ebbe forse vita non breve) è stata coliocata nel X-XI sec.. I materiali ceramici dello strato di profanazione della chiesa triabsidata di Castel Tirolo sembrano sostanzialmente affini ai materiali dello scavo citato. Come non troppo dissimili del resto sembrano anche taluni frammenti provenienti dal riempimento della tomba 3, la quale risulta essere stata coperta e sigillata da tutti e tre i diversi e successivi pavimenti della chiesa triabsidata e dunque è anteriore alla costruzione della medesima. Va inoltre tenuto presente che tra la fase di demolizione dell'edificio e lo scavo del fossato ebbe il tempo di depositarsi sopra la rovina un pacco di detriti di pendio di spessore rilevante.

${ }^{10}$ [Geochron] gx/20003/ams : 1055 +- 55 b. [ETH] ..........660/886, (datazioni calibrate). Si è tenuta presente l'ipotesi che potessero provenire dall'originaria struttura lignea del tetto della chiesa triabsidata il cui riutilizzo nell'ambito della ricostruzione tarda appare verosimile, ma naturalmente potrebbero appartenere in toto a questa fase di ricostruzione.

${ }^{11}$ Cfr. STEINMANN BRODTBECK, 1939; inoltre E. PÖSCHEL 1954; inoltre O. SCHÄFER—R. SENNHAUSER 1966; SENNHAUSER 1979; infine RÜBER SCHÜTTE 1992; Eadem 1993.

${ }^{12}$ Cfr. GAMBER, 1970; inoltre GLEIRSCHER-NOTHDURFTER, 1987, particolarmente pp. 284-294. 
${ }^{13}$ Cfr. STEINMANN BRODTBECK, 1939, p. 84; ALPAGO NOVELLO, 1969.

${ }^{14}$ Ringrazio per questa segnalazione Ivan Matejčić della Soprintendenza ai Beni Culturali dell'Istria.

${ }^{15}$ Cfr. RÜBER SCHÜTTE, 1993, particolarmente p. 111.

${ }^{16}$ Cfr. DESCHAMPS, 1954, p. 255; inoltre HASELOFF, 1980 p. 38; inoltre RASMO, 1981 pp. 32-33.

${ }^{17} \mathrm{Cfr}$. GLEIRSCHER-NOTHDURFTER 1987, p. 294.

${ }^{10}$ Cfr. STEINMANN BRODTBECK, 1939, p. 82; NOTHDURFTER 1990, pp. 152-153. Merita comunque di essere riferita la tradizione locale secondo cui la chiesa sarebbe stata costruita dall'imperatrice Theophanu, sposa di Ottone II nell'anno 981 (GUFLER, 1986, p. 9)

${ }^{19}$ Cfr. NOTHDURFTER, 1990, pp. 154-155.

${ }^{20}$ Non sono stati effettuati scavi e la datazione rimane incerta (cfr. RASMO, 1982, p. 46).

${ }^{21}$ Cfr. RASMO, 1985 p. 18. Per indicazioni sui recenti scavi nella parrocchiale di Civezzano presso Trento, ringrazio i colleghi Gianni Ciurletti e Gianni Rizzi.

${ }^{22}$ Cfr. HUBL, 1982, p. 323.

${ }^{23}$ Sul significato magico religioso del ferro di cavallo in contesti funerari cfr. CAPITANIO, 1980.

24 Il contenuto dello scrigno comprendeva tre distinti involti con frammenti di ossá, capelli (?) e materiale nerastro simile a pece. Si sono ritrovati minutissimi resti di uno scritto su carta (papiro?), con ogni probabilità un documento di autenticazione delle reliquie stesse. Di fatto neppure una lettera era distintamente decifrabile. Si tratta in ogni caso delle tracce del più antico documento cartaceo finora noto nel territorio dell'antico Tirolo. Potrebbe trattarsi di reliquie di santi divenuti "scomodi" in quanto storicamente legati a posizioni divenute eretiche, nel corso di dispute teologiche di particolare gravità (per questa ed altre indicazioni ringrazio I. Rogger del Museo Diocesano di Trento). Altri ipotizzano invece una lacuna nella fruizione dell'edificio sacro (cfr. NOTHDURFTER, 1996, pp. 607-608). La cappella attuale di Castel Tirolo è dedicata a San Pancrazio.

${ }_{25}$ R. Sennhauser, colloca l'iscrizione, preliminarmente in un ampio arco di tempo tra VI-VIII secolo, con maggiori probabilità per l'VIII secolo. Non presenta problemi la formula "in Christi nomine hic requiescit" e l'aggettivo finale "albada" che si riferisce con ogni probabilità ad una particolare condizione della defunta (da tradurre forse con "senza peccato"), analogamente ad es. al termine "inox" dell'iscrizione di Radoara, venuta in luce nell'edificio termale di Kaiseraugst. Notevoli problemi presenta invece la lettura del nome (femminile) del personaggio qui sepolto (o semplicemente qui ricordato).

${ }^{26}$ Cfr. DAL RI-RIZZI, 1995, pp. 89-90. Inoltre figg. 7 e 8. Inoltre WACKERNELL, 1996, p. 584.

${ }^{27}$ Si può ipotizzare per la conca di Merano il trasferimento di un potente locale, in una fase caratterizzata da mutate situazioni strategiche, dal Castrum Maiense situato in prossimità del fondovalle a controllo di una strada, verso il colle dove sorgerà secoli dopo Castel Tirolo, sicuramente più naturalmente munito per quanto concerne le necessità di difesa e più dominante nell'intenzione di un'esibizione di potenza.

${ }^{28}$ Cfr. MAIER, 1989 p. 186

${ }^{29}$ Cfr. LUNZ, 1978.

${ }^{30}$ Cfr. DAL RI, 1993.

${ }^{31}$ Cfr. SEEBACHER, 1995

${ }^{32}$ Cfr. BOSCHI-CIURLETTI, 1978, pp. 341, 346, 347, tavole V, X, XIII.

${ }_{33} \mathrm{Nel}$ caso dello stipite di sinistra, per ottenere l'altezza sufficiente è stato necessario inserire nella parte superiore un pilastrino alto circa $40 \mathrm{~cm}$ con dei motivi decorativi che stilisticamente non si adeguano a quelli delle parti inferiori, con palesi tracce di un adattamento secondario (rilavorazione dei bordi). Naturalmente anche una provenienza di tutte o di parte di queste spoglie dalla primitiva cappella di Castel Tirolo, le cui tracce (abside) sono visibili al di sotto dell'attuale, non è a priori da escludere.

${ }^{34}$ Cfr. TAGLIAFERRI, 1981, p. 312 (nr. 324), tav, CXLIV.

${ }^{35}$ Cfr. RASMO, 1982, p. 47, fig. 45. Per il fregio con grappoli cfr. PASSAMANI, 1992, p. 273, fig. 213. Per il capitello Meccorona, cfr. nota 41.

${ }^{36}$ Cfr. RASMO, 1976, p. 149, fig. 147; IDEM, 1981, pp. 10-13, 38-39; IDEM 1985, pp. 17-18; cfr. anche SPADA, 1981, pp. 41-78.

${ }^{37}$ Cfr. ORSI, 1883, p. 147 (Vignola di Arco); cfr. inoltre ATZ, 1909, fig. 33-35 (Tenno, Riva del Garda), fig. 62 a p. 104 (pluteo dalla chiesa parrocchiale di Santa Maria a Bolognano, attualmente al Museo Civico di Rovereto); CECCHELLI, 1928 (Stenico, Premione, Seo, Lundo, Vigo Lomaso); RASMO, 1976, pp. 154-155, inoltre IDEM 1982, pp. 39-47 (varie località trentine); CAVADA, 1992, p. 31, fig. 3 (Santa Maria di Tione).

${ }^{38}$ PONTALTI, 1989.

${ }^{39}$ Cfr. GEROLA, 1926 (Dos Trento); BOSCHI_CIURLETTI 1977 (Santa Maria Maggiore); RASMO 1976, particolarmente pp. 153-154, fig. 156; IDEM 1982, p. 44 fig. 42,43 (basilica vigiliana).

${ }^{10}$ Cfr. RASMO, 1982, pp. 45-46.

"Cfr. DAL RI-ROSSI, 1987, pp. 240-241, fig. 20-21 (probabilmente dalla chiesa di Santa Maria Assunta).

${ }^{12}$ EGGER, 1930, pp. 329-330, fig. 3; RASMO, 1985, p. 18 (Sabiona; San Candido); BIERBRAUER-NOTHDURFTER, 1988 , tav. 17 (Sabiona).

${ }^{43}$ Cfr. RASMO, 1976, p. 153; IDEM 1981, p. 10.

${ }^{44}$ Cfr. KARPF, 1995, p. 68. Colpisce in ogni caso che proprio uno dei centri più importanti dell' attività missionaria dei duchi Agilolfingi e cioè il convento di San Candido, fondato nel 776 da Tassilo III, di fatto non abbia restituito quasi traccia di scultura carolingia (cfr. RASMO, 1980) mentre nel territorio carinziano da conventi benedettini coevi di identica origine sono note abbondanti testimonianze di questa classe di evidenze (cfr.: IDEM, p. 72-73, tanto che sculture a treccia di quest'epoca sono considerate da questo autore una caratteristica peculiare delle chiese sorte nell'ambito di tale attività di missione.

${ }^{15}$ Cfr. da ultimo FOGLIARDI, 1992.

${ }^{46}$ In particolare per i reperti carolingi di Stenico, da ultimo cfr. CAVADA, 1996, pp. 31-35.

${ }^{47}$ Vi si dedica attualmente Paola Porta dell'Università di Bologna.

${ }^{43}$ Sui ritrovamenti tardo antichi e altomedievali di Sanzeno cfr. GHISLANZONI, 1931, particolarmente nota (1) a p. 410. Inoltre GEROLA, 1933; BONFANTI-DAL RI, 1983; infine SIRONI, 1989 e ROGGER, 1989. Si è di recente richiamata l'attenzione (ROGGER, 1989, particolarmente pp. 339-341) 
su significativi ritrovamenti che furono effettuati in passato nell'ambito della chiesa di Sanzeno. Nel 1472 al momento della costruzione della chiesa attuale "sub altari vetusto in loco dirupto" o secondo un'altra fonte "sub altari cori veter is ecclesiae" fu rinvenuta una capsella d'argento "in qua inter cineres recondita fuerunt quaedam ossa ... sanctorum martirum"; da un'altra fonte sappiamo che tali resti erano "parvo sub marmore clausi" (MENAPACE, 1986). Rogger (che dubita per parte sua che il luogo del martirio sia da identificare senz'altro con l'attuale parrocchiale di Sanzeno e che le reliquie ritrovate a suo tempo abbiano realmente una stretta relazione con i Martiri), distingue opportunamente tra le reliquie (frammenti di ossa) conservate nella minuscola teca (nel frattempo scomparsa) ed il grosso del materiale rinvenuto (ceneri, carboni, intonaco, semi carbonizzati, frantumi di legno). La descrizione dei materiali raccolti assieme alle ossa tra i resti del rogo (ceneri, etc.), ricorda singolarmente le evidenze caratteristiche di un Brandopferplatz di tradizione retica. Interessante è la stretta correlazione in questo caso di Sanzeno in base alle fonti scritte, con Saturno, divinità assai diffusa in queste aree in epoca romana, sicuramente un'antica divinità locale fatta oggetto di interpretatio (cfr. PASCAL, 1964). E' noto che le pratiche di culto nei Brandopferplätze che durante l'antico e medio impero sembravano essere scomparse (ma che più probabilmente sopravvissero nascoste dietro i culti ufficiali), conobbero nel IV secolo un nuovo momento di fortuna, come dimostrato dai ritrovamenti in regione, tra l'altro al Burgstall del Monte Sciliar in Alto Adige e inoltre al Piller Sattel nel Tirolo settentrionale. Colpisce cioè l'analogia tra l'evidenza dei Brandopferplätze, particolarmente numerosi in territorio trentino, altoatesino e nordtirolese dove è documentato l'uso d'incenerire le vittime (animali, ma anche esseri umani) su rozzi altari e la sorte subita dai tre missionari inviati dal vescovo Vigilio che furono bruciati dinanzi al tempio di Saturno ("in cospectu idoli veteris Saturni tempestate longeva") cfr. lettera di Vigilio vescovo di Trento a San Giovanni Crisostomo; e ancora "preparatus est de sacris ecclesiae culminibus sive trabibus robus. Haec martires flamma velavit" cfr. lettera a San Sempliciano, vescovo di Milano (cfr. ROGGER, 1989).

${ }^{49}$ Cfr. RASMO 1982, p. 42. Questo autore indica possibili analogie iconografiche con le due lastre triangolari con figurazioni di pavoni da San Salvatore di Brescia. Per un'immagine fotografica dei frammenti di pilastrino ottagonale, cfr. FAUSTINI, 1939, p. 193. Pilastrini ottagonali, in questo caso lisci, sono presenti a San Benedetto di Malles, cfr. RÜBER, 1991, p. 136. Pilastrini in parte analoghi decorati su tutte le facce sono noti da Brescia, San Salvatore (?) (cfr. PANAZZA-TAGLIAFERRI, 1966, fig. 92, tav. XXXI, pp. 86-87).

${ }^{50}$ Per l'ambone dalla basilica ariana di Ravenna, cfr. FARIOLI CAMPANATI, 1987. p. 250. fig. 3a.

${ }^{5}$ Cortese indicazione di E. Cavada dell'Ufficio Beni Archeologici di Trento e di C. Salizzoni di Ponte Arche.

${ }^{52}$ Il Bognetti ipotizza che qui si trovasse il piccolo monastero in cui si ritirò per morire "apud Tridentum" l'abate Secondo di Non (Hist. Long. IV, 40; cfr. BOGNETTI, p. 325).

\section{BIBLIOGRAFIA}

AA.VV., 1978, San Salvatore di Brescia. Materiali per un museo I e II, Brescia

ALPAGO-NOVELLO, A., 1969, Influenze bizantine ed orientali nel Veneto settentrionale (in relazione ad alcuni monumenti inediti), in "Archivio Storico di Belluno, Feltre e Cadore" XL, pp. 81-95.

ARSLAN, E., 1954, L'architettura dal 568 al 1000 in "Storia di Milano" II, pp. 499-608.

ATZ, K., 1909, Kunstgeschichte Tirols und Vorarlbergs

BIERBRAUER, V., NOTHDURFTER, HANS, 1988, Die Ausgrabung im spätantik-frühmittelalterlichen Bischofssitz Sabiona-Säben, in "Der Schlern” 62, pp. 243-300.

BOGNETTI, G.P., 1954, Sul tipo e il grado di civiltà dei Longobardi in Italia secondo i dati dell'archeologia della storia dell'arte, Arte nell'Alto Medioevo nella regione alpina, Atti del III congresso internazionale per lo studio dell'Alto Medioevo 9-14 settembre 1951.

BOGNETTI, G.P., 1967, I loca sanctorum e la storia della chiesa nel regno dei longobardi, in "L'età longobarda", 3, Milano, pp. $303-346$.

BOGYAY, TH., 1957, Zum Problem der Flechtwerksteine. Karolingische und Ottonische Kunst. Werden-Wesen-Wirkung in "Forschungen, Kunstgeschichte und Christliche Archäologie" 3, Wiesbaden, pp. 262-276.

BONFANTI POL, M., DAL RI, L., 1986, Un antico sarcofago cristiano da Sanzeno in Val di Non, Scritti in onore di Nicolò Rasmo, Bolzano, pp. $59-75$.

BOSCHI, R., CIURLETTI, G., 1980, Il ritrovamento della ecclesia intra civitatem a Trento; contributo allo studio dei rapporti tra lapicidi longobardi e il Trentino, Atti del $6^{\circ}$ Convegno Internazionale di Studi sull'Alto Medioevo, Milano 21-25 ottobre 1978, Spoleto, pp. 329-339.

BRAUN, J., 1924, Der christliche Altar in seiner geschichtlichen Entwiklung, München

CAMINADA, CHR., 1956, Die Skulpturen im Schloß Tirol ikonographisch und volkskundlich gedeutet, in "Zeitschrift für Schweizerische Archäologie und Kunstgeschichte", 16, pp. 140-156.

CAPITANIO, M. A., 1981, Chiodi e ferri da cavallo nel folclore europeo. Notizie edite ed inedite, in "Archivio per l'Antropologia el'Etnologia" Vol. CXI, pp. 235-245.

CASAGRANDE, V., 1926, La Diocesi di Trento e i suoi antichi monumenti eucaristici, Congresso eucaristico della Diocesi Tridentina, pp. 20.

CASARTELLI-NOVELLI, S., 1974, Corpus della scultura altomedievale VI, La diocesi di Torino, Spoleto

CAVADA, E., 1992, Testimonianze di età romana e tardoantica nelle Giudicarie interne (Trentino occidentale). Aggiornamenti, in "Judicaria" 19-20, pp. 29-48.

CAVADA, E., 1996, Preesistenze edilizie nell'area della cappella, in G. Fogliardi, Le pitture murali delle cappella di San Martirio nel castello di Stenico, Beni Artistici e Storici del Trentino, Quaderni 4, pp. 22-35.

CECCHELLI, C., 1928, Reliquie trentine dell'età barbarica, in "Studi trentini di Scienze Storiche", anno IX, fasc. III, pp. 193-212.

CECCHELLI, C., 1954, Pittura e scultura carolingia in Italia, in "Problemi della civiltà carolingia", Spoleto, pp. 193 ss.

CHEVALIER, P., 1995, L'ambon à Poreč et en Istrie, in "Hortus artium medievalium, Journal of the International Research Center for Late Antiquity and Middle Ages", vol. 1. Zagreb-Motovun, pp. 126-133.

CHINI, E., 1987, L'arte nelle Giudicarie Esteriori, in AA.VV. Le Giudicarie Esteriori. Banale, Bleggio, Lomaso. cultura e storia, Trento, pp. 1-101. 
CHIERICI, G., 1942, Le sculture della basilica di San Michele Maggiore a Pavia, Milano.

CLAVADETSCHER, U., JANOSA, M., 1987, Die Ausgrabungen in der Pfarrkirche St. Vincentius in Pleif bei Vella, in "Bündner Monatsblatt" 9/10, Chur, pp. 275-292.

CODROiCO, R., POMPEATI MARTINI, M., PONTALTI, F., 1983, Beni Culturali del Trentino. Monumenti, Chiesa di Santa Maria del Pernone o del Perdono a Varone, Trento, pp. 20-21.

COLETTI, L., 1978, L'arte nel territorio di Concordia dal Medioevo al Rinascimento, Julia Concordia dall'età romana all'età moderna, Treviso, pp. 210305.

COURVOISIER, H.R., SENNHAUSER, R.H., 1996, Die Klosterbauten - eine Übersicht in Müstair, Kloster - St. Johann, Vol. I zur Klosteranlage Vorklösterliche Befunde, Veröffentlichungen des Instituts für Denkmalpflege an der ETH/Zürich, Vol. 16.1, pp. 15-65.

DAL RI, L., 1989(91), Gli edifici medievali dello scavo di Piazza Walther a Bolzano, Bolzano dalle origini alla distruzione delle mura, Atti del Convegno Bolzano aprile 1989, pp. 245-303.

DAL RI, L., 1993, Ausgrabungen des Denkmalamtes Bozen in St. Stephan ob Burgeis (Gemeinde Mals) und Sankt Laurentius in Kortsch (Gemeinde Schlanders), Der Vinschgau und seine Nachbarräume (Edit, R. Loose), pp. 5i-64.

DAL RI, L., RIZZI, G., 1995, Il territorio altoatesino alla fine del VI e nel VII secolo d. C. Città, castelli, campagne nei territori di frontiera (secoli VI e VII), $5^{\circ}$ Seminario sul Tardoantico el'Altomedioevo nell'Italia centrosettentrionale, Monte Barro-Galbiate (Lecco) 9-10 giugno 1994, Documenti di Archeologia, 6, pp. 87-113.

DAL RI, L., ROSSI, G., 1987, Antichi sarcofagi cristiani a Mezzocorona nel Trentino, Kunst und Kirche in Tirol. Festschrift zum 70. Geburtstag von Karl Wolfsgruber, pp. 219-251.

DALLA BARBA BRUSIN, D., 1964, Scultura ad intreccio altomedievale a Grado, Memorie Storiche Forogiuliesi, vol, XLV, pp. $172-178$.

DANNHEIMER, H., 1980, Steinmetzarbeiten der Karolingerzeit. Neufunde aus altbayerischen Klöstern, Ausstellungskatalog der Prähistorischen Staatssammlung, München, 6 .

DANNHEIMER, H., 1986, Zur Rekonstruktion der Chorschranken von Mals, Scritti in onore di Nicolò Rasmo (a cura di S. Spada), pp. 93-102.

DANNHEIMER, H., 1988, Kirchenbau, Zur Ausstattung der Kirchen, Die Bajuwaren. Von Severin bis Tassilo 488-788, Catalogo della Mostra, pp. 299310 .

DAVIS WEYER, C., 1987, Müstair, Milano e l'Italia carolingia, in "Il millennio ambrosiano", Milano, pp. 202-237.

DE FRANCOVICH, G., 1945, Arte carolingia ed ottoniana in Lombardia, in "Römisches Jahrbuch für Kunstgeschichte", pp. 113-255.

DEFUNS, A., 1996, Tumelg/Tomils, Sogn Murezi, in "Jahresbericht 1995 des archäologischen Dienstes Graubünden und der Kantonalen Denkmalpflege Graubünden”, pp. 122-124.

DEGASPERI, F., 1990, Lacerti altomedievali della zona del Basso Sarca, in "Il Sommolago", VII-n.3, pp. 101-116.

DESCHAMPS, P., 1954, A propos de pierres à décor d'entrelacs et de stucs de Saint-Jean de Mustair, in Arte dell'alto Medioevo nella regione alpina. Actes du IIIe Congrès international pour l'étude du haut Moyen Age, 9-14 septembre 1951, pp. 253-268.

DOBERER, E., 1962, Zur Bestimmung frühmittelalterlicher Werkstücke, in "Österreichische Zeitschrift für Kunst und Denkmalpflege" 16, pp. 86-88.

DOBERER, E., 1965, Die ornamentale Steinskulptur an der karolingischen Kirchenausstattung, Karl der Große Lebenswerk und Nachleben, (W. Braunfels ed.), vol. III, p. 203-253.

EGG, E., 1973, Kunst in Tirol, Innsbruck.

EGGER, A., 1930, Sabiona, die erste Heimat der Diözese, in "Der Schlern" 11, Heft 6, pp. 223-230.

EGGER, R., 1916, Frühchristliche Kirchen im südlichen Norikum, Sonderschriften des österreichischen archäologischen Instituts in Wien IX, Wien.

ERHARD, B., 1995, Zur Baugeschichte von Schloß Tirol. Zu einer Zusammenfassung, Eines Fürsten Traum. Meinhard der II. Das Werden Tirols. Tiroler Landesausstellung, pp. 64-67.

FARIOLI CAMPANATI, R., 1986, La scultura architettonica e di arredo liturgico a Ravenna alla fine della tarda antichità. I rapporti con Costantinopoli Cristiana, Storia di Ravenna, pp. 249-267.

FAUSTINI, G., 1989, La Storia, Splendore d'Anaunia, Trento, 1989, pp. 135-241.

FILLITZ, H., 1958, Die Spätphase des “langobardischen” Stiles, in "Jahrbuch kunsthistorischer Sammlungen Wien” 54, pp. 7-72.

FOGLIARDI, G., 1987, I frammentialtomedievali inseriti nell'abside romanica di San Lorenzo a Tenno, significato primario e traslato, in "Studi Trentinti di Scienze Storiche" Sez. II-1, pp. 5-39.

FORLATI TAMARO, B., 1956, Il duomo di Vicenza. Ritrovamenti e scoperte, Vicenza.

GAMBER, J, 1970, Churrätische Saalkirchen mit Dreiapsiden-Chor liturgiegeschichtlich untersucht, in "Römische Quartalschrift für Christiliche Altertumskunde und Kirchengeschichte 65, pp. 98-126.

GARBER J., 1915, Die karolingische St. Benediktkirche in Mals, in "Zeitschrift des Museums Ferdinandeum" 59, pp. 3-61.

GEROLA G., 1926, I monumenti antichi del Dos Trento, in “Trentino. Rivista della legione Trentina”, anno II, nr. 9, settembre, pp. $205-212$.

GEROLA, G., 1933, Nuove scoperte nella chiesa di Sanzeno, in "Studi Trentini di Scienze Storiche" XIV, p 179.

GEROLA, G., 1933B, Le scoperte di archeologia cristiana a Sanzeno. Tracce evidenti di una necropoli romana, in "Il Brennero" del 11.06 .1933$.

GHISLANZONI, E., 1931, Sanzeno nell'Anaunia, Scavi di abitazioni preromane, Regione X (Venetia et Histria), Notizie degli Scavi, vol. VII, pp. $409-471$. GINHART, K., 1942, Die karolingische Flechtwerksteine in Kärnten, Aus den römischen in Germanischen Kärntern, Festschrift für R. Egger, Klagenfurt, pp.180-235.

GINHART, K, 1942, Die karolingische Flechtwerksteine in Kärnten, in “Carinthia”, I, 132, pp. 112-167.

GINHART, K., 1954, Karolingische und frühromanische Werkstücke in Kärnten, in "Carinthia I" 144, pp. 205-243. 
GLASER, F., 1989, Das Münster in Molzbichl, das älteste Kloster Kärntens, in "Carinthia l" 179, pp. 99-124.

GLASER, F., 1993, Neu entdeckte Flechtwerksteine in Kärnten, in "Carinthia I" 183, pp. 319-330.

GLASER, F., 1996, Kirchenbau und Gotenherrschaft. Auf den Spuren des Arianismus in Binnennorikum und in Rätien II, in "Der Schlern" 70, fas. 2, pp. 83-100.

GLEIRSCHER, P., NOTHDURFTER, H., 1987, Die Kirchengrabungen von St. Georg bei Völlan, Lana, in “Der Schlern”. 61, fasc. 2, pp. $267-305$.

GOLL, J., MARTI, S., KERSTEN, W., WOHLGEMUTH, M., 1996, Eine Abguß-Sammlung für Hans Rudolf Sennhauser, Katolog des frühnittelalterlichen Plastik, in "Georges-Bloch-Jahrbuch des Kunstgeschichtlichen Seminars der Universität Zürich, pp. 57-116, 227-275 (Mals "Italien, St. Benedikt: Chorschrankenplatte und Kapitell" di S. Demetz, p. 100).

GOTTER, .., Das Schloss Tyrol, 1968, in "Mitteilungen der K.K. Central-Commission" XIII, pp. XLII-XLIII.

GRAY, N., The paleography of latin inscription in the Eigth, Ninth, Tenth Centuries in Italy, in "Papers of the British School at Rome" 14-16, 1938-48 4346, pp. 38-171.

GRUBER, K., 1971, Südtiroler Heiligen-Himmel. Namenspatrone in der heimischen Kunst, Bolzano.

GUFLER, Chr., 1986, St. Margarethenkirche in Lana, Laurin Kunstführer nr. 105, Bolzano.

HASELOFF, A., 1930, Die vorromanische Plastik in Italien, Firenze-Berlin.

HASELOFF, A., 1980, Die frïhmittelalterlichen Chorschrankenfragmente in Müstair, in "Helvetia Archaeologica" 11, pp. 21-35.

HÖLDL, G., 1993, Karantanien und der Alpen-Adria-Raum im Frühmittelalter (Wien-Köln-Weimar), (Grabmayer, J. Ed.),, pp. 37-99.

HUBERT, J., 1968, L'impero Carolingio, Milano.

HUBERT, J., PORCHER J., VOLBACH, W.F., 1969, Die Kunst der Karolinger, München.

JACOBSEN, W., SCHÄFER, L., SENNHAUSER, A.R., 1991, Vorromanischen Kirchenbauten. Katalog der Denkmäler bis zum Ausgang der Ottonen. Nachtragsband (ed. Zentralinstitut für Kunstgeschichte, München).

JOHANNSON-MEERY, B., 1993, Karolingerzeitliche Flechtwerksteine aus dem Herzogtum Baiern und aus bayerisch-Schwaben, in "Katalogen Prähistorischer Sammlung" 27, Kallmünz/Opf. 1993.

JURKOVIĆ, M., 1995, Il ciborio di Novigrad (Cittanova d'Istria), in "Hortus artium medievalium. Journal of the International Research Center for Late Antiquity and Middle Ages" vol. 1. Zagreb-Motovun, p. 141-149.

KARAMAN, L., 1932, Notes sur l'art byzantin et les Slaves catholiques de Dalmatie, in “L'art byzantin chez les Slaves” II, pp. 332-380.

KARPF, K., 1995, Steinerne Kirschenanstattungen in Karantanien. Zur Problematik frühmittelalterlicher Flechtwerksteine, in Symposium zur Geschichte Millstatt und Kärnten 16. und 17. Juni 1995 (F. Nikolatsch ed.), pp. 66-79.

KAUTZSCH R., 1939, Die römische Schmuckkunst in Stein vom 6. bis 10. Jahrhundert, in "Römisches Jahrbuch für Kunstgeschichte" 3, 3-73.

KAUTZSCH, R., 1941, Die langobardische Schmuckkunst in Oberitalien, in "Römisches Jahrbuch für Kunstgeschichte" 5, pp. 1-48.

LORENZONI, G., 1974, Monumenti di età carolingia. Aquileia, Cividale, Malles, Müstair, Padova.

LUNZ, R., 1978, Frühmittelalterliche Stuckornamente von St. Peter bei Meran, Archäologisch- Historische Forschungen in Tirol, Beiheft 1.

MARUŠIĆ, B., 1979, Conoscenza della scultura altomedievale in Istria, Museo Archeologico d'Istria-Pola, Guida III, Pula.

MAYR, M., 1899, Die Erbauung des Schlosses Tirol und die Grïndung des Klosters Steinach bei Meran, in "Zeitschrift des Ferdinandeums", 43, pp.181213.

MENAPACE, G.B., 1896, Intorno al luogo del martirio dei tre santi anauniesi, in "L'Anaunia Sacra" Anno I. N. 6, 29 Ottobre 1896, pp.185-360.

MENIS, G., 1976, La basilica paleocristiana nelle regioni alpine orientali, in "Antichità Altoadriatiche" 9.

MÜLLER, I., 1986, Die Frühzeit des Klosters Disentis, Forschungen und Grabungen, in "Bündner Monatsblatt", pp. 1-45

MÜLLER, TH., 1935, Mittelalterliche Plastik Tirols, Berlin.

NICOLUSSI, K., 1995, Schloß Tirol-Dendrochronologische Erkenntnisse zur Baugeschichte. Jahrringe von Hölzer als neue Geschichtsquelle. Eines Fürsten Traum. Meinhard der II. Das Werden Tirols. Tiroler Landesausstellung, pp. 74-78.

NOLL, R., 1954, Frühes Christentum in Österreich, Wien.

NOTHDURFTER, H., 1990, Lana; Kirchengrabungen als Geschichtsquellen, in "100. Jahre Lana", pp.148-176.

NOTHDURFTER, H., 1996, Anstelle einer Rezension, in "Der Schlern" 70, fasc. 10, pp. 603-608.

ONGARI, D., 1992, Ricerche sul luogo del martirio di San Vigilio nella chiesa di Spiazzo Rendena, pp. 3-39.

ORSI, P., 1883, Monumenti cristiani anteriori al mille, in "Archivio Storico per Trieste, L'Istria e il Trentino", pp. 129-148.

OSWALD, F., SCHÄFER, L., SENNHAUSER, H.R., 1966, Vorromanische Kirchenbauten. Katalog der Denkmäler bis zum Anfang der Ottonen, Veröffentlichungen des Zentralinstituts für Kunstgeschichte 3, München.

PANAZZA, G., TAGLIAFERRI, A., 1966, Corpus della scultura altomedievale III. La diocesi di Brescia, Spoleto.

PASCAL, C.B., 1964, The Cults of Cisalpine Gaul, Collection Latomus.

PASSAMANI, B., 1962, Ignoti resti di scultura trentina, in "Studi Trentini di Scienze Storiche", pp. 379-382.

PAULI L., 1979(80), Die Alpen in Frühzeit und Mittelalter. Die archäologische Entdeckung einer Kulturlandschaft, München.

PAULUS, H., 1956, Kleiner Katalog karolingischer Flechtwerksteine, in "Nachrichten des Deutschen Instituts für Merowingisch-Karolingischen Kunstforschungen" I2, pp 1-8 + Tavole 1-21.

PERGULA, P., 1987, La chiesa ed il monastero di San Calocero fuori le mura ad Albenga: relazione preliminare sulle campagne di scavo 1985-1986, in "Archeologia in Liguria" III.2, pp. 445-454.

PERONI, A., 1972, La plastica in stucco nel S. ambrogio di Milano. Arte ottoniana e romanica in Lombardia. Kolloquium über spätantike und frühmittelalterliche Skulptur, Universität Heidelberg, Institut für Ur- und Frühgeschichte, pp. 59-111. 
PERONI, A., 1976, Per la tipologia architettonica dell'età carolingia nell'area lombarda, Roma e l'età carolingia, Atti delle giornate di studio 3-8 maggio 1976. Istituto Nazionale di Archeologia e Storia dell'arte, pp. 87.

PERONI, A, 1986, Stucco e pittura nel S. Benedetto di Malles, Scritti in onore di Nicolò Rasmo, pp. 79-89.

PETRICIOLI, I., 1995, Sculpture in Zadar between the late roman and pre-romanesque periods, in "Hortus artium medievalium. Journal of the International Research Center for Late Antiquity and Middle Ages" vol. 1. Zagreb-Motovun, p. 74-82.

PFISTER, F., 1954, s.v. Brandeum, Reallexikon für Antike und Christentum (Th. Klauser editore) Vol. II, pp. 522-523.

PIGHI, G.B., 1963, Verona nell'VIII secolo, Verona.

POESCHEL, E., 1937 (1975), Die Kunstdenkmäler des Kantons Graubünden, I.

POESCHEL, E., 1954, Frühchristliche und frühmittelalterliche Architektur in Churrätien, Arte dell'Alto Medioevo nella Regione Alpina, Atti del III Congresso internazionale per lo Studio dell'Alto Medioevo, 9-14 settembre 1951, pp. 119-132.

RASMO N., 1976, Problemi di arte longobarda e carolingia nella regione atesina, Roma e l'età carolingia. Atti delle giornate di Studio 3-8 maggio 1976, a cura dell'Istituto di Storia dell'Arte dell'Università di Roma, pp. 148-158.

RASMO, N., 1953, La scultura romanica nell'Alto Adige, in "Cultura Atesina"7, pp. 9-49, Tavv. I-XI.

RASMO, N., 1957, La basilica paleocristiana di Bolzano, in "Cultura Atesina" XI, pp. 7-20, Tavv. VII-VIII.

RASMO, N., 1961A, Architettura medievale nel Trentino e nell'Alto Adige.

RASMO, N., 1961B, Costruzioni altomedievali in Anaunia, in "Beiträge zur Kunstgeschichte und Archäologie des Frühmittelalters", p. 196 segg.

RASMO, N., 1970, Castel Tirolo, in "Cultura Atesina" IX.

RASMO, N., 1973, Südtirol-Trentino.

RASMO, N., 1981, Arte carolingia nell'Alto Adige, Bolzano.

RASMO, N., 1982, Storia dell'Arte nel Trentino, Trento.

RASMO, N., 1985, L'Alto Adige nell'Arte, Rosenheim.

RODRIGUEZ, H., Bemerkungen zur relativchronologischen Gliederung der südalpinen spätrömisch- spätantiken Gebrauchskeramik. Il territorio tra tardo antico e altomedioevo. Metodo di indagine e risultati, $3^{\circ}$ Seminario sul Tardo Antico e l'Alto Medioevo nell'area alpina e padana. Monte Barro Galbiate (Como) 9-11 settembre 1991, Biblioteca di archeologia Medievale, 9, pp. 159-178.

ROGGER, I., 1967, 1968, 1973, 1974, Scavi e ricerche sotto la cattedrale di Trento, in "Studi Trentini di Scienze Storiche" Annata XLVI fasc. I, pp. 197-212 + Tavole I-VI, Annata XLVII fasc. I, pp. 3-26, Annata LII fasc. I, pp. 375-392, Annata LIII fasc. I, pp. 387-409.

ROGGER, I., 1989, Interessi agiografici del vescovo Hinderbach, con particolare riguardo al santorale trentino, Il principe vescovo Johannes Hinderbach (1465-1486) fra Medioevo e Umanesimo, Atti del Convegno promosso dalla Biblioteca Comunale di Trento 2-6 ottobre 1989, pp. 319-380.

ROMANINI, A. M., 1988, Il Medioevo. Storia dell'Arte Classica e italiana (dir. G.C. Argan), Firenze.

ROSCHMANN, A., 1756, Inscriptiones et alia diversi generis romana per omnem Tirolim monumenta (Manoscritto inedito).

RÜBER, E., 1991, St. Benedikt in Mals, Europäische Hochschulschriften Reihe XXVIII Kunstgeschichte, Vol. 130, Frankfurt a.M.

RÜBER-SCHÜTTE, E., 1993, Neue Forschungen zu St. Benedikt in Mals, Der Vinschschgau und seine Nachbarräume. Vorträge des landeskundlichen Symposiums veranstaltet vom Südtiroler Kulturinstitut Schloß Goldrain, 27 bis 30 Juni 1991, pp. $71-81$ (Rainer Loose editore).

RUGO, P., 1975, Le iscrizioni dei sec. VI-VII-VIII esistenti in Italia, Vol. II, Venezia e Istria.

SAGE, W., 1988, Kirchenbau 1. zu Typen und Bauweise, Die Bajuwaren. Von Severin bis Tassilo 488-788, Catalogo della Mostra, pp. $293-299$.

SANDBERG, G., 1977, Bistum Chur in Südtirol. Untersuchungen zur Ostausdehnung ursprünglicher Hochstiftsrechte im Vintschgau, in "Zeitschrift für bayerische Landesgeschichte", 40, Heft 2/3, pp. 706-825.

SANNAZARO, M., 1994; Materiali archeologici paleocristiani e altomedievali, Carta Archeologica della Lombardia IV, La provincia di Lecco, pp. 284318.

SCHAFFRAN, E., Funde aus der Völkerwanderungszeit in Museum zu Trient, II, Funde aus Stein, in "Mitteilungen der Deutschen Akademie in München" $\mathrm{XV}$, pp. 458-465.

SCHINKE, RUTH, 1992, Romanische Portale auf Schloß Tirol, Bolzano.

SCHMÖLZER, H., 1901, Notizen Nr. 36, in "Mitteilung̈en der Central Kommission für Denkmalpflege" serie II, vol. 27, Wien, pp. $54-55$.

SEEBACH, G., 1995, Die romanischen Portale auf Burg Tirol - Eine bauhistorische Untersuchung, Eines Fursten Traum Meinhard der II. Das Werden Tirols, Tiroler Landesausstellung, pp. 79-92.

SENNHAUSER, R. H., 1979, Spätantike und frühmittelalterliche Kirchen Churrätiens, Von der Spätantike zum frühen Mittelalter, (a c. d. J. Werner), in "Vorträge und Forschungen" 25, Sigmaringen, pp. 193-218.

SIRONI, E., 1989, Dall'Oriente in Occidente: $i$ santi Sisinio, Martirio e Alessandro morti in Anaunia, Sanzeno.

SPADA PINTARELLI, S., 1981, Frammenti di marmo, di stucco e di affresco di San Benedetto di Malles (catalogo), Arte carolingia in Alto Adige, (N. Rasmo Editore), Bolzano, pp. 41-78.

SPADA PINTARELLI, S., Frammenti carolingi della parrocchiale di Bolzano, Bolzano dalle origini alla distruzione delle mura. in "Atti del convegno di studi organizzato dall'Assessorato alla Cultura del Comune di Bolzano", Castel Mareccio, aprile 1989, pp. 143-148.

STEINMANN-BRODTBECK, S., 1939, Herkunft und Verbreitung des Dreiapsidenchores, in "Zeitschrift für Schweizerische Archäologie und Kunstgeschichte" 1, 65-95.

SULSER, W. 1954, Die St. Luziuskirche in Chur, Arte dell'alto Medioevo nella regione alpina, in "Actes du IIIe Congrès international pour l'étude du Moyen Age", 9-14 September 1951, pp. 151-166.

SYDOW V., W., 1985, Religion und Kulte der Kaiserzeit in Rom und im Gebiet des heutigen Tirol, Veldidena, Römisches Militärlager und Zivilsiedlung, catalogo della mostra, pp. 137-145.

SYDOW V., W., 1986, Die frühchristliche Kirche von Ampaß und ihre Nachfolgebauten, in "Veröffentlichungen des Tiroler Landesmuseum Ferdinandeum", vol. 66, Innsbruck, p. 71-126.

SZAMEIT, E., 1994, Merowingisch-karantanisch-awarische Beziehungen im Spiegel archäologischer Bodenfunde des 8. Jahrhunderts - Ein Beitrag zur Frage nach den Wurzeln frïhmittelalterlicher Kulturerscheinungen im Ostalpenraum, in "Neues aus Alt-Villach" 31, pp. 7-23.

TAGLIAFERRI, A., 1981, Corpus della scultura altomedievale, vol. X, La diocesi di Aquileia e Grado. 
TIELLA, M., 1969, San Clemente di Castione, in "Atti della Accademia Roveretana degli Agiati", Anno Accademico 217, Serie VI, Vol. VII, Fasc. A, 1967, pp. 115-133.

UBL, H., 1982, Frühchristliches Österreich, Severin zwischen Römerzeit und Völkerwanderungen, Catalogo della Mostra, Enns, pp. 295-336.

ULBERT, T., 1969, Studien zur dekorativen Reliefplastik des östlichen Mittelmeerraumes (Schrankenplatten des 4.-10. Jahrhunderts), Miscellanea Byzantina Monacensia, X, pp.1-69 tavv, 1-23.

ULBERT, T., 1988, Zur liturgisch-funktionellen Ausstattung spätantiker Kirchen des Alpenraums, Die Bajuwaren. Von Severin bis Tassilo 488-788. Catalogo della Mostra, pp. 287-292.

VEDOVELLI, G., 1990, Le sculture murate nelle pareti della chiesa di Santa Maria a Cisano di Bardolino, Il Garda, l'ambiente, l'uomo, VI miscellanea di Studi. Centro Studi per il Territorio Benacense, pp. 43-62.

VERZONE, P., 1942, L'architettura religiosa dell'Alto Medioevo nell'Italia Settentrionale, Milano.

Wackernell, N., 1996, Der römische Brückenpfeiler in Algund und die Römerstraße Claudia Augusta im Etschtal und Burgrafenamt, in "Der Schlern" 70 , fasc. 10, pp. 594-602.

WEBER, S., 1912, Antichi monumenti eucaristici nel Trentino, in "Rivista Tridentina", a. XII, fasc. 3.

ZOVATTO, P.L., 1964, L'arte altomedioevale, Verona e il suo territorio, II, Verona, pp. 479-582.

ZULIANI F., L'Alto Medioevo 2, I marmi di San Marco. Uno studio ed un catalogo della scultura ornamentale marciana fino all'XI secolo, Venezia.

\title{
SVJEDOČANSTVA O SAKRALNIM GRAĐEVINAMA KAROLINŠKOG I OTONSKOG RAZDOBLJA U POKRAJINI ALTO ADIGE. ISKOPAVANJA U CASTEL TIROLO
}

\author{
SAŽETAK
}

Arheološkim istraživanjima Soprintendenze Provinciale dell'Alto Adige, kojima je tijekom posljednjeg desetljeća obuhvaćeno nekoliko sakralnih građevina, obogaćena su svjedočanstva o karolinškom razdoblju. To ponajprije vrijedi za nedavno vođeno iskopavanje lokaliteta Castel Tirolo kod Merana, postojbine dinastije grofova Tirolskih. U rujnu 1992. prigodom redovitih radova na vanjskom plaštu obrambenog bedema otkriveni su ostaci stare crkve o kojoj se nisu sačuvali nikakvi podaci. Iskopavanja koja su uslijedila 1994. godine nastavljena su u najnovije doba.

Postojanje tri upisane apside $\mathrm{u}$ istočnom zidu crkve omogućile su vezivanje ove građevine s tipom "rätische Dreiabsidenkirchen" (recijske tropsidalne crkve), podrobnije proučavanim na prostoru kantona Grigioni (Sennhauser, 1979.). Crkva je naposlijetku bila srušena i sravnjena sa zemljom, vjerojatno uslijed gradnje kaštela koji je imao svoju vlastitu kapelicu (San Pancrazio). Ta se gradnja odnedavno, na temelju dendrokronološkog podatka, datira u XII. stoljeće (1138/9.). Glede uništene sakralne građevine, radiokarbonska datacija greda koje su izgorjele u vremenu napuštanja crkve dala je u ovom slučaju godine $895 \pm 55$ (Geochron Laboratories 14.12.94.), odnosno 660-886. (ETH Z, rich). Po svojoj shemi crkva koja se nalazi unutar prostora pokrajine Alto Adige, blisko podjeća na crkve kao što su Santa Margherita i San Giorgio, obje u obližnjoj općini Lana i na kapelu u Castel Appiano. Za gradnju nedavno istražene troapsidalne crkve San Giorgio predložena je datacija unutar prve polovice X. stoljeća (Gleirscher Nothdurfter, 1987.).

Nastavak radova unutar crkve pokazao je da je karolinško zdanje počivalo na temeljima jednoapsidalne građevine iz V-VI. stoljeća, od koje je otkriven izvorno zazidan i zapečaćen mramorni grob s relikvijama, s intaktnim relikvijarom od pozlaćenog srebra.

Brežuljak na kojem je podignut Castel Tirolo smješten je u Meranskoj kotlini, na ulazu u Val Venosta. U toj dolini nalazi se crkva San Benedetto di Malles, analogne strukture, opće poznata već od početka ovog stoljeća.

U istoj općini, u selu Burgusio, 1989. i 1991. godine arheološki je istražena crkva Santo Stefano, grobna crkva benediktinskog samostana Marienberg. U ovom slučaju ustanovljeno je kako je današnja građevina preuzela u suštini nepromijenjenu karolinšku shemu. Posrijedi je dvoetažna crkva s kvadratnim svetištem koja počiva na temeljima starokršćanske crkve, zasigurno povećane u VII. stoljeću. Prigodom drugih novijih istraživanja crkvenih građevina utvrđeno je da starokršćanska zdanja nisu u karolinškom razdoblju pretrpjela spomena vrijedne strukturalne promjene. Takav je slučaj i s crkvom San Virgilio al Virgolo u Bolzanu, izvorno najvjerojatnije kapelom kastruma $\mathrm{Bau}$ zanum, kojeg 690. godine spominje Pavao Đakon. Karolinška faza ovdje je zastupljena opremom pronađenom u jednom grobu, dočim je ziđe ostalo nepromijenjeno u odnosu na građevinu V-VI. stoljeća. Radikalne promjene zgrada je doživjela tek u IX. stoljeću.

Složeniji je primjer (stolne) crkve Uznesenja Marijina u Bolzanu za koju je Nicolò Rasmo (1957.) ustanovio da je u karolinškom razdoblju doživjela osjetno smanjenje širine tlocrta u odnosu na izvornu starokršćansku strukturu. Ovoj je fazi pripisana i nekolicina sekundarno pronađenih ulomaka oslikane žbuke (Spada, 1991.).

Glede plastike karolinškog razdoblja, spominjemo na kraju skupinu nalaza iz crkve sv. Marije u gradu Trento, jedinoj urbanoj cjelini trajnog karaktera na pripadajućem teritoriju. Iz pokrajine Alto Adige potječu, naprotiv, već desetljećima poznate štukature i mramorni reljefi crkve San Benedetto di Malles. Na obližnjem teritoriju Trenta, u crkvi Sanzeno u Val di Non (moguće je da je riječ o kastrumu Aniaunumkojeg navodi Pavao Đakon) prigodom nedavnih restauratorskih radova na vidjelo su došli ostaci skuptura vrlo fine fakture, možda pilastrići pluteja, sekundarno - kao građevinski materijal — upotrijebljeni pri gradnji romaničkog zvonika. 